\title{
DEFINING METRIC SPACES VIA OPERATORS FROM UNITAL $C^{*}$-ALGEBRAS
}

\author{
BRANKA PAVLOVIĆ
}

For a unital $C^{*}$-algebra $A$ and an operator $T$ with $\operatorname{Dom} T \subseteq$ $A$, Range $T$ in a normed space, and $\operatorname{ker} T=\mathbb{C}$ mathrm1, we consider the metric $d_{T}$ on $\mathcal{S}(A)$, the state space of $A$, given by $d_{T}(\phi, \psi)=\sup \{|\phi(a)-\psi(a)|: a \in A \&\|T a\| \leq 1\}$, for $\phi, \psi \in \mathcal{S}(A)$. This is a generalization of the definition given by A. Connes for defining a metric on $\mathcal{S}(A)$ via unbounded Fredholm modules over $A$.

The main problem of our investigation, posed by M. Rieffel, is the relationship between thus defined metric topology $\mathcal{T}_{d_{T}}$, and the weak-* topology $\mathcal{T}_{w^{*}}$ on $\mathcal{S}(A)$. We give two different complete characterizations of those operators for which $\mathcal{T}_{d_{T}}=$ $\mathcal{T}_{w^{*}}$. First, we establish the relevance to this relationship of the induced one-to-one operator $\hat{T}: \operatorname{Dom} T / \mathbb{C} 1 \rightarrow$ Range $T$, and $B_{1}=\{a \in \operatorname{Dom} T:\|T a\| \leq 1\} / \mathbb{C} 1$, which is the inverse image under $\hat{T}$ of the unit ball of RangeT. We show that: (1) $d_{T}$ is bounded if and only if $B_{1}$ is bounded, if and only if $\hat{T}^{-1}$ is bounded; (2) $\mathcal{T}_{d_{T}}=\mathcal{T}_{w^{*}}$ if and only if $\overline{B_{1}}$ is compact, if and only if $\hat{T}^{-1}$ is compact. Furthermore, we consider the de Leeuw derivation $D_{d_{T}}$ associated to $T$, which is defined by $(f(y)-f(x)) / d_{T}(x, y), x, y \in \mathcal{S}(A)$, and is an operator from $C(\mathcal{S}(A))$ into $C_{b}(Y), Y=\{(x, y) \in \mathcal{S}(A) \times \mathcal{S}(A): x \neq y\}$, whose domain is the Lipschitz algebra $\operatorname{Lip}\left(\mathcal{S}(A), d_{T}\right)$. We show that $\mathcal{T}_{d_{T}}=\mathcal{T}_{w^{*}}$ if and only if $D_{d_{T}}$ is unbounded on every infinite dimensional subspace of its domain. In particular, we use all these results to characterize those unbounded Fredholm modules over $A$ whose metric topology coincides with the weak-* topology on $\mathcal{S}(A)$.

\section{Introduction.}

It is well established to think of $C^{*}$-algebras as "noncommutative" topological spaces. The idea to define a "noncommutative metric space" using $C^{*}$-algebraic tools was initiated by A. Connes [2] and [3]. He suggests that the right notion of a noncommutative metric is that of an unbounded Fredholm module, and proposes the following definition. 
Definition 1.1. Let $A$ be a unital $C^{*}$-algebra. An unbounded Fredholm module $(\mathcal{H}, D)$ over $A$ is:

(C1): a Hilbert space $\mathcal{H}$ which is a left $A$-module, that is, a Hilbert space $\mathcal{H}$ and a $*$-representation of $A$ on $\mathcal{H}$;

(C2): an unbounded, self-adjoint operator $D$ on $\mathcal{H}$ such that $\{a \in A$ : $[D, a]$ is densely defined and extends to a bounded operator on $\mathcal{H}\}$ is norm dense in $A$;

(C3): $\left(1+D^{2}\right)^{-1}$ is a compact operator (i.e. $D$ has compact resolvent).

Connes shows ([2], Proposition 4) that if $A$ is any unital $C^{*}$-algebra and $(\mathcal{H}, D)$ an unbounded Fredholm module over $A$ such that

(C4): $\{a \in A:\|[D, a]\| \leq 1\} / \mathbb{C} 1$ is bounded, then $d$, defined for $\phi, \psi \in \mathcal{S}(A)$, the state space of $A$, by the formula

$$
d(\phi, \psi)=\sup \{|\phi(a)-\psi(a)|: a \in A,\|[D, a]\| \leq 1\},
$$

defines a metric on $\mathcal{S}(A)$. Condition (C3) is not needed for this.

Connes also shows ([2], Proposition 1) that if $M$ is a compact, spin, Riemannian manifold, $A=C(M), \mathcal{H}=L^{2}(M, S)$, and $D$ the Dirac operator, then the geodesic distance $d(P, Q)$, for $P, Q \in M$ is given by

$$
d(P, Q)=\sup \{|a(P)-a(Q)|: a \in A,\|[D, a]\| \leq 1\},
$$

so that the two notions of metric coincide.

M. Rieffel [11] poses the following question: For which compact metric space $(X, d)$ there exists an unbounded Fredholm module over $C(X)$ such that $d$ is defined by (2). He shows that it is true for every compact metric space if the condition (C3) is dropped from the definition of the unbounded Fredholm module, but he notes that in that case we need some additional non-degeneracy conditions.

We follow a similar path here, but in the opposite direction, which was as a problem also posed by M. Rieffel. Namely, suppose that the metric defined by an unbounded Fredholm module is given. The problem we address here is the relationship of the metric and the weak-* topology on the state space. To cope with the problem, we have to consider a more general situation, as follows.

When we have a triple $(A, \mathcal{H}, D)$, where $(\mathcal{H}, D)$ is an unbounded Fredholm module over a unital $C^{*}$-algebra $A$, then $\mathcal{B}(\mathcal{H})$ is a Banach $A$-module, and the map $T: A \rightarrow \mathcal{B}(\mathcal{H})$ defined by $T a=[D, a]$, where $D$ is as in $(\mathrm{C} 2)$, is a densely defined derivation from $A$ into $\mathcal{B}(\mathcal{H})$, and the metric given by the formula (1) is

$$
d_{T}(\phi, \psi)=\sup \{|\phi(a)-\psi(a)|: a \in A \&\|T a\| \leq 1\},
$$

for any $\phi, \psi \in \mathcal{S}(A)$, the state space of $A$. As a generalization, we let $T$ be any operator with $\operatorname{Dom}(T) \subseteq A$ and $\operatorname{Range}(T) \subseteq B$, where $B$ is any normed space, and we let $d_{T}$ be defined by (3). 
Here is a brief description of the results presented in this paper. We start by considering the commutative case first (Sections 2, 3, 4, and 5), when $A=C(X), X$ a compact Hausdorff space, so that $X$ is homeomorphic to the pure state space of $A$. Even more generally, sometimes we want to consider $A=C_{b}(X)$, the algebra of all bounded continuous functions on $X$, where $X$ is any topological space. As we are really interested in producing a metric on $X$ so that the metric topology on $X$ coincides with the original topology on $X$, we are really interested only in metrizable spaces. However, some of our results are valid even when we consider $X$ to be a general topological space.

We first search for conditions which $T$ should satisfy so that $d_{T}$ is really a metric on $X$ (Section 2). In particular, we characterize those operators from $A=C_{b}(X)$ which define a bounded metric on $X$ (Theorem 2.2).

Next, we explore the relationship of the metric topology $\mathcal{T}_{d_{T}}$ and the original topology $\mathcal{T}$ on $X$ (Section 3). We show that under reasonable assumptions, the topologies are comparable, and in fact, the metric topology is finer. This is always so when $X$ is compact Hausdorff, and the metric also turns out to be always complete, so that $\mathcal{T}_{d_{T}}=\mathcal{T}$ if and only if $\left(X, d_{T}\right)$ is compact (Proposition 3.1). However, even when $X$ is compact Hausdorff, $\mathcal{T}_{d_{T}}$ need not be locally compact, which we show by example (Remark 3.2).

In Section 4, we turn our attention to Lipschitz algebras and de Leeuw derivations. We show how for every operator which defines a metric on $X$, we obtain the de Leeuw derivation associated to that operator, the one which defines the same metric on $X$ (Proposition 4.5). By using the results of J. Johnson [5], we show that $(X, d)$ is precompact if and only if $D_{d}$, the de Leeuw derivation defined by $d$, is unbounded on every infinite dimensional subspace of its domain (Theorem 4.6). We also show, following M. Rieffel [11], how the de Leeuw derivations are connected to unbounded Fredholm modules (Remark 4.8).

We conclude the commutative case with necessary and sufficient conditions for the metric topology $\mathcal{T}_{d_{T}}$, and the original topology $\mathcal{T}$ on $X$ to coincide (Section 5). We present two different sets of conditions: (1) $\mathcal{T}_{d_{T}}$ $=\mathcal{T}$ if and only if $\overline{\{f \in \operatorname{Dom}(T):\|T f\| \leq 1\} / \mathbb{C} 1}$ is compact (Corollary 5.2); (2) in terms of associated de Leeuw derivation $D_{d_{T}}$ we have that $\mathcal{T}_{d_{T}}=\mathcal{T}$ if and only if $D_{d_{T}}$ is unbounded on every infinite dimensional subspace of its domain (Theorem 5.3). It follows that for $\mathcal{T}_{d_{T}}=\mathcal{T}$, it is necessary that $T$ is unbounded on every infinite dimensional subspace of its domain. However, we show that this is not a sufficient condition (Example 5.7), by using some results about spaces $c_{0}$ and $l_{1}$ from [7].

Finally, we extend all these results to the noncommutative situation (Section 6), by using the canonical (or Kadison's) function representation $\Phi$ of a unital $C^{*}$-algebra $A$ into $C(\mathcal{S}(A))$, where $\mathcal{S}(A)$ is the state space of $A$. For 
each commutative theorem we obtain its noncommutative analog (Proposition 6.2, Theorem 6.3, and Theorem 6.4). Furthermore, we show that if an operator separates points of $\mathcal{S}(A)$, and we know that it defines an appropriate metric (bounded, or $\mathcal{T}_{d_{T}}=\mathcal{T}_{w^{*}}$ ) on $\overline{\mathcal{P}(A)}$, the pure state space of $A$, then it defines such a metric on all of $\mathcal{S}(A)$ (Theorem 6.5). In particular, that is always so when $\overline{\operatorname{Dom}(T)}=A$. In commutative case, that says that the metric extends from $X$ to the metric on $\mathcal{P}(X)$, the space of all the probability measures on $X$, which has the same properties (Corollary 6.6). Finally, we use all these results to characterize those unbounded Fredholm modules over $A$ which define a bounded metric on $\mathcal{S}(A)$, or a metric whose topology coincides with $\mathcal{T}_{w^{*}}$ on $\mathcal{S}(A)$ (Theorem 6.8).

At the end, it is interesting to note that the conditions for $\mathcal{T}_{d_{T}}=\mathcal{T}_{w^{*}}$ are not algebraic, as we expected when we started working on the problem, and thus worked with derivations. Instead, one should consider any operator $T$, and the conditions involve the topology on $\operatorname{Dom}(T)$ given by the norm of the algebra $A \supseteq \operatorname{Dom}(T)$. It is precisely due to this fact - that conditions for $\mathcal{T}_{d_{T}}$ $=\mathcal{T}_{w^{*}}$ do not use the algebraic structure - that we can extend all the results from the commutative to the noncommutative case (Section 6), by using the canonical function representation, which is a bicontinuous operator, but not a homomorphism of the algebra.

As a conclusion, we note that although Theorem 6.8 characterizes those unbounded Fredholm modules over a unital $C^{*}$-algebra $A$ for which the metric and the weak-* topology coincide, the condition (C3) (that $\left(1+D^{2}\right)^{-1}$ is compact) is not used. In [10] we will explore the influence of that condition, as well as the $p$-summability of $D$ (see $[\mathbf{2}]$ ), on the metric topology. We also point out that Lipschitz algebras appeared once again naturally when discussing metric spaces. This relationship, and the importance of the de Leeuw derivation is further explored in [9]. In particular, it is shown that the category in which the objects are compact metric spaces and the morphisms are Lipschitz maps is (contravariantly) equivalent to the category in which the objects are Lipschitz algebras and the morphisms are homomorphisms. This category is also characterized in terms of de Leeuw derivations. This is analogous to the equivalence of the category in which the objects are compact Hausdorff spaces and the morphisms are homeomorphisms and the category in which the objects are unital commutative $C^{*}$-algebras and the morphisms are ${ }^{*}$-homomorphisms.

\section{Defining a metric by an operator.}

We begin with a general discussion of the conditions which an operator from $C(X)$ (where $X$ is any metrizable space) should satisfy in order to define a metric on $X$. For completeness and for easy reference we recall the "classical" definition of a metric. 
Definition 2.1. A metric on a set $X$ is a function $d: X \times X \rightarrow \mathbb{R}^{+}$which satisfies the following conditions. For $x, y, z \in X$ :

(M1): $d(x, y)=0 \Leftrightarrow x=y$ (definiteness);

(M2): $d(x, y)=d(y, x)$ (symmetry);

(M3): $d(x, y)+d(y, z) \geq d(x, z)$ (triangle inequality).

Let $(X, \mathcal{T})$ be a topological space and let $B$ be a normed space. Let $T: \operatorname{Dom}(T) \rightarrow B$ be an operator with $\operatorname{Dom}(T) \subseteq C_{b}(X)$. Let a function $d_{T}: X \times X \rightarrow \mathbb{R}^{+} \cup\{\infty\}$ be defined by

$$
\begin{aligned}
d_{T}(x, y) & =\sup \{|f(x)-f(y)|: f \in \operatorname{Dom}(T) \&\|T f\| \leq 1\} \\
& =\sup \{|f(x)-f(y)| /\|T f\|: f \in \operatorname{Dom}(T)\},
\end{aligned}
$$

for $x, y \in X$. We say that $d_{T}$ is the metric defined by operator $T$.

Obviously $d_{T}(x, y)=d_{T}(y, x)$ for all $x, y \in X$, i.e. condition (M2) holds. Also, $d_{T} \geq 0$, and $d_{T}(x, x)=0$ for all $x \in X$. Furthermore, $d_{T}$ satisfies the triangle inequality (M3). To see that, let $x, y, z \in X$. If $d(x, y)=\infty$ or $d(y, z)=\infty$ then (M3) is clearly satisfied. Otherwise

$$
\begin{aligned}
& d_{T}(x, y)+d_{T}(y, z) \\
= & \sup \{|f(x)-f(y)|:\|T f\| \leq 1\}+\sup \{|g(y)-g(z)|:\|T g\| \leq 1\} \\
= & \sup \{|f(x)-f(y)|+|g(y)-g(z)|:\|T f\| \leq 1,\|T g\| \leq 1\} \\
\geq & \sup \{|f(x)-f(y)|+|f(y)-f(z)|:\|T f\| \leq 1\} \\
\geq & \sup \{|f(x)-f(z)|:\|T f\| \leq 1\}=d_{T}(x, z) .
\end{aligned}
$$

To have the other inclusion of (M1) satisfied, notice that $d_{T}(x, y)=0$ implies $x=y$ if and only if

(O1): $\operatorname{Dom}(T) \subseteq C_{b}(X)$ separates points of $X$.

For $d_{T}(x, y)=0$ if and only if $|f(y)-f(x)|=0$ for all $f \in \operatorname{Dom}(T)$ with $\|T f\| \leq 1$, so in fact for all $f \in \operatorname{Dom}(T)$.

The only other requirement which needs to be satisfied is that $d(x, y)<\infty$ $\forall x, y \in X$. Let 1 denote the constant function $1(x)=1 \forall x \in X$. For $d(x, y)<\infty$ it is necessary that $\operatorname{ker}(T) \subseteq \mathbb{C} 1$. We now show that we may assume that $\operatorname{ker} T \supseteq \mathbb{C} 1$ and so $\operatorname{ker} T=\mathbb{C} 1$. Suppose that $1 \in \operatorname{Dom}(T)$ and $T 1=b_{1} \neq 0$. Then $B_{1}=B / \mathbb{C} b_{1}$ is a normed space with the norm $\|[b]\|=$ $\inf \left\{\left\|b+b_{1} c\right\|: c \in \mathbb{C}\right\}$, for $b \in B$. Let $S: B \rightarrow B_{1}$ be the quotient map $S b=$ $[b], b \in B$. Define operator $T_{1}: \operatorname{Dom}\left(T_{1}\right) \rightarrow B_{1}$ with $\operatorname{Dom}\left(T_{1}\right)=\operatorname{Dom}(T)$ by $T_{1} f=S T f=[T f], f \in \operatorname{Dom}(T)$. We now show that $d_{T_{1}}(x, y)=d_{T}(x, y)$ for all $x, y \in X$. Since $\left\|T_{1} f\right\|=\inf \left\{\left\|T f+b_{1} c\right\|=\|T(f+c)\|: c \in \mathbb{C}\right\}$ $\leq\|T f\|$ we have that $\|T f\| \leq 1$ implies $\left\|T_{1} f\right\| \leq 1$, i.e. $\{f:\|T f\| \leq 1\} \subseteq$ $\left\{f:\left\|T_{1} f\right\| \leq 1\right\}$. Thus $d_{T_{1}}(x, y) \geq d_{T}(x, y)$ for any $x, y \in X$. To show the opposite inequality, let $\varepsilon>0$. For each $f \in \operatorname{Dom}\left(T_{1}\right)$ with $\left\|T_{1} f\right\| \leq 1$ there exists $c \in \mathbb{C}$ such that $\|T(f+c)\|<\left\|T_{1} f\right\|+\varepsilon$, and if $g=(f+c) /(1+\varepsilon)$, we have $\|T g\| \leq 1$ and $|g(x)-g(y)|=|f(x)-f(y)| /(1+\varepsilon)$. Thus by taking 
supremum over all such $f$, we have $d_{T}(x, y) \geq d_{T_{1}}(x, y) /(1+\varepsilon)$, and thus $d_{T}(x, y) \geq d_{T_{1}}(x, y)$. This shows that we may and will from now on always assume that

\section{(O2): $\operatorname{ker} T=\mathbb{C} 1$.}

Note that we have only the necessary condition for $d_{T}(x, y)<\infty$. In the case when $d_{T}$ is supposed to be a bounded metric on $X$, we have conditions which are both necessary and sufficient.

Theorem 2.2. Let $(X, \mathcal{T})$ be a topological space, and let $B$ be a normed space. Let $T$ be a linear operator with $\operatorname{Dom}(T) \subseteq C_{b}(X)$, and Range $(T) \subseteq$ $B$, which satisfies: $(\mathrm{O} 1) \operatorname{Dom}(T)$ separates points of $X$; and $(\mathrm{O} 2) \operatorname{ker} T=$ $\mathbb{C} 1$. Let $d$ be defined by $(4) ;$ let $d_{X}=\sup \{d(x, y): x, y \in X\}$; let for $z \in X$, $M(z)=\{f: f(z)=0\}$; let $Q$ denote the quotient operator $Q: \operatorname{Dom}(T) \rightarrow$ $\operatorname{Dom}(T) / \mathbb{C} 1$; and let $\hat{T}$ be the induced one-to-one operator $\operatorname{Dom}(T) / \mathbb{C} 1 \rightarrow$ $B$. The following are equivalent:

(M4) $d$ is a bounded metric on $X$.

(O31) $\{f \in \operatorname{Dom}(T):\|T f\| \leq 1\} / \mathbb{C} 1$ is bounded. Equivalently, $\hat{T}^{-1}$ is bounded.

(O32) For any fixed $z \in X,\{f \in \operatorname{Dom}(T): f \in M(z) \&\|T f\| \leq 1\}$ is bounded. Equivalently, $\left(\left.T\right|_{M(z)}\right)^{-1}$ is bounded.

Furthermore: (1) if $d_{X}<\infty$, then $\left\|\hat{T}^{-1}\right\| \leq d_{X}$ and $\left\|\left(\left.T\right|_{M(z)}\right)^{-1}\right\| \leq d_{X}$; (2) if $\left\|\hat{T}^{-1}\right\|<\infty$, then $\left\|\left(\left.T\right|_{M(z)}\right)^{-1}\right\| \leq 2\left\|\hat{T}^{-1}\right\|$ and $d_{X} \leq 2\left\|\hat{T}^{-1}\right\|$; (3) if $\left\|\left(\left.T\right|_{M(z)}\right)^{-1}\right\|<\infty$, then $\left\|\hat{T}^{-1}\right\| \leq\left\|\left(\left.T\right|_{M(z)}\right)^{-1}\right\|$ and $d_{X} \leq 2\left\|\left(\left.T\right|_{M(z)}\right)^{-1}\right\|$.

Proof. As usual, the norm on $\operatorname{Dom}(T) / \mathbb{C} 1$ is $\|Q f\|=\inf \{\|f+c\|: c \in \mathbb{C}\} \leq$ $\|f\|$.

$(\mathrm{O} 31) \Rightarrow(\mathrm{M} 4)$ We show that $d_{X} \leq 2\left\|\hat{T}^{-1}\right\|$. Let $x, y \in X$. For any $f \in \operatorname{Dom}(T)$ with $\|T f\| \leq 1$, and any $c \in \mathbb{C},|f(x)-f(y)|=\mid(f(x)+c)-$ $(f(y)+c)|\leq| f(x)+c|+| f(y)+c \mid \leq 2\|f+c\|$. So $|f(x)-f(y)| \leq$ $2 \inf \{\|f+c\|: c \in \mathbb{C}\}=2\|Q f\| \leq 2\left\|\hat{T}^{-1}\right\|$, and $d(x, y)=\sup \{|f(x)-f(y)|:$ $f \in \operatorname{Dom}(T) \&\|T f\| \leq 1\} \leq 2\left\|\hat{T}^{-1}\right\|$. In particular, $d_{X} \leq 2\left\|\hat{T}^{-1}\right\|$.

$(\mathrm{M} 4) \Rightarrow(\mathrm{O} 31)$ Conversely, suppose that $d_{X}<\infty$. Consider any $f \in$ $\operatorname{Dom}(T)$ with $\|T f\| \leq 1$. Since $|f(x)-f(y)| \leq d(x, y) \leq d_{X}$ for any $x, y \in$ $X$, we see that with $c=f(x)$ for some fixed $x \in X$, and $g=f-c$, we have $\|g\|_{\infty} \leq d_{X}$, and hence $\|Q f\| \leq d_{X}($ in $C(X) / \mathbb{C} 1)$. So $\left\|\hat{T}^{-1}\right\| \leq d_{X}$.

$(\mathrm{O} 31) \Longleftrightarrow(\mathrm{O} 32)$ It is clear that $\left.Q\right|_{M(z)}$ is one-to-one onto, and that $\|f\| \geq\|Q f\|$. In fact, $\left.Q\right|_{M(z)}$ is bicontinuous. Let $f \in \operatorname{Dom}(T) \cap M(z)$, let $c \in \mathbb{C}$, and let $g=f+c$. Then $g(z)=c$, so $\|g\| \geq|c|$. On the other hand, $\|g\| \geq|\|f\|-| c||$, so $\|g\| \geq \max \{|c|,|\|f\|-| c||\} \geq\|f\| / 2$. This shows that $\|Q f\| \geq\|f\| / 2$, which is the same as $\left\|\left(\left.Q\right|_{M(z)}\right)^{-1}\right\| \leq 2$. The equivalence of (O31) and (O32) is immediate. 
Showing that $d_{X} \leq 2\left\|\left(\left.T\right|_{M(z)}\right)^{-1}\right\|$ and that $\left\|\left(\left.T\right|_{M(z)}\right)^{-1}\right\| \leq d_{X}$ is as in $(\mathrm{O} 31) \Leftrightarrow$ (M4). Also, $\left\|\hat{T}^{-1}\right\|=\left\|Q\left(\left.T\right|_{M(z)}\right)^{-1}\right\| \leq\|Q\|\left\|\left(\left.T\right|_{M(z)}\right)^{-1}\right\| \leq$ $\left\|\left(\left.T\right|_{M(z)}\right)^{-1}\right\|$, and $\left\|\left(\left.T\right|_{M(z)}\right)^{-1}\right\|=\left\|\left(\left.Q\right|_{M(z)}\right)^{-1} \hat{T}^{-1}\right\| \leq\left\|\left(\left.Q\right|_{M(z)}\right)^{-1}\right\|\left\|\hat{T}^{-1}\right\|$ $\leq 2\left\|\hat{T}^{-1}\right\|$.

The condition (O31) is derived from the condition (C4) of Connes. In fact, it implies also that $\operatorname{ker} T \subseteq \mathbb{C} 1$. In particular, if $(X, \mathcal{T})$ is compact Hausdorff, and the metric topology is expected to coincide with $\mathcal{T}$, then $d_{T}$ should be bounded, and so $T$ should satisfy condition (O31).

Remark 2.3. We conclude this section by showing that if the operator $T$ is closable, then $d_{\bar{T}}=d_{T}$, where $\bar{T}$ is the closure of $T$. Let $(X, \mathcal{T})$ be a metrizable space, and let $B$ be a normed space. Let $T$ be a closable linear operator with $\operatorname{Dom}(T) \subseteq C_{b}(X)$, and Range $(T) \subseteq B$. Let $d_{\bar{T}}$ and $d_{T}$ be defined by (4). Since $\{f \in \operatorname{Dom}(T):\|T f\| \leq 1\} \subseteq\{f \in \operatorname{Dom}(\bar{T}):\|\bar{T} f\| \leq 1\}$, it is clear that $d_{\bar{T}} \geq d_{T}$. To show the reverse inequality, let $x, y \in X$. For any $f \in \operatorname{Dom}(\bar{T})$ with $\|\bar{T} f\| \leq 1$, and for any $\varepsilon>0$, there exists $g \in \operatorname{Dom}(T)$ such that $\|T g\| \leq 1,\|g-f\|<\varepsilon / 2$, and $\|T g-\bar{T} f\|<\varepsilon / 2$. So $|f(x)-f(y)|$ $\leq|f(x)-g(x)|+|g(x)-g(y)|+|g(y)-f(y)|<|g(x)-g(y)|+\varepsilon$. In particular, since this is true for any $\varepsilon,|f(x)-f(y)| \leq \sup \{|g(x)-g(y)|: g \in$ $\operatorname{Dom}(T) \&\|T g\| \leq 1\}=d_{T}(x, y)$. So $d_{\bar{T}}(x, y) \leq d_{T}(x, y)$, which is what we wanted to show.

\section{Metric and weak-* topologies.}

We further explore the relationship between $\mathcal{T}_{d_{T}}$, the topology on $X$ induced by the metric $d_{T}$ defined by an operator $T$, and $\mathcal{T}$, the original topology on $X$. We are also interested in some general properties of the metric $d_{T}$.

Let $(X, \mathcal{T})$ be a topological space, and let $B$ be a normed space. Let $T$ be a linear operator with $\operatorname{Dom}(T) \subseteq C_{b}(X)$ which separates points of $X$, Range $(T) \subseteq B$, and $\operatorname{ker} T=\mathbb{C} 1$, so that $T$ defines a possibly infinite metric $d_{T}$ on the set $X$. Let $\mathcal{T}_{C_{b}(X)}$ be the weak topology on $X$ induced by $C_{b}(X)$, let $\mathcal{T}_{\operatorname{Dom}(T)}$ be the weak topology on $X$ induced by $\operatorname{Dom}(T)$, and let $\mathcal{T}_{d_{T}}$ be the topology on $X$ induced by $d_{T}$. The following relationship holds among these topologies: $\mathcal{T} \supseteq \mathcal{T}_{C_{b}(X)} \supseteq \mathcal{T}_{\operatorname{Dom}(T)}$ and $\mathcal{T}_{d_{T}} \supseteq \mathcal{T}_{\operatorname{Dom}(T)}$.

We show the last inclusion, $\mathcal{T}_{d_{T}} \supseteq \mathcal{T}_{\operatorname{Dom}(T)}$. Let $f \in \operatorname{Dom}(T)$ with $\|T f\|=$ 1 , and let $V$ be an open subset of $\mathbb{C}$. Let $U=\{x \in X: f(x) \in V\}$, so that $U$ is in the subbase of the topology $\mathcal{T}_{\operatorname{Dom}(T)}$. For any $x \in U$ there exists $r>0$ such that $\mathbb{D}(f(x), r)=\{z \in \mathbb{C}:|f(x)-z|<r\} \subseteq V$. Then $B_{d_{T}}(x, r)=\left\{y \in X: d_{T}(x, y)<r\right\} \subseteq U$. For if $y \in B_{d_{T}}(x, r)$, then $|g(x)-g(y)|<r$ for all $g \in \operatorname{Dom}(T)$ with $\|T g\| \leq 1$, and in particular $|f(x)-f(y)|<r$, so that $f(y) \in \mathbb{D}(f(x), r) \subseteq V$, and $y \in U$. 
It is easy to see that if $\overline{\operatorname{Dom}(T)}=C_{b}(X)$, which is most often the case, then $\mathcal{T}_{C_{b}(X)}=\mathcal{T}_{\operatorname{Dom}(T)}$. So under reasonable assumptions we have $\mathcal{T}=$ $\mathcal{T}_{C_{b}(X)}=\mathcal{T}_{\operatorname{Dom}(T)}$, and in that case $\mathcal{T}_{d_{T}} \supseteq \mathcal{T}$, that is, $\mathcal{T}_{d_{T}}$ is finer then $\mathcal{T}$, and in particular, it means that these topologies are comparable.

In the special case of our interest, $(X, \mathcal{T})$ is compact Hausdorff, and we have the following direct result.

Proposition 3.1. Let $(X, \mathcal{T})$ be a compact Hausdorff space, and let $B$ be a normed space. Let $T$ be a linear operator with $\operatorname{Dom}(T) \subseteq C(X)$ which separates points of $X$, Range $(T) \subseteq B$, and $\operatorname{ker} T=\mathbb{C} 1$, so that $T$ defines a possibly infinite metric $d$ on the set $X$. Then:

(a) $\mathcal{T}_{d}$ is finer than $\mathcal{T}$, that is $\mathcal{T} \subseteq \mathcal{T}_{d}$;

(b) the metric space $(X, d)$ is complete;

(c) $\mathcal{T}_{d}=\mathcal{T}$ if and only if $(X, d)$ is compact.

Proof. (a) Let $U$ be any open set in $\mathcal{T}$. It is enough to show that $\forall x \in$ $U \exists r>0$ such that $B(x, r)=\{y \in X: d(x, y)<r\} \subseteq U$. Fix any $x \in U$. The set $Y=X \backslash U$ is compact in $\mathcal{T}$. Since $\operatorname{Dom}(T)$ separates points of $X$, for any $y \in Y$ there exists $f_{y} \in \operatorname{Dom}(T)$ such that $f_{y}(x)=0$ and $\left|f_{y}(y)\right|>1$. The set $U_{y}=\left\{z \in X:\left|f_{y}(z)\right|>1\right\}$ is open in $\mathcal{T}$. The family of open sets $\left\{U_{y}\right\}_{y \in Y}$ covers $Y$, so there is a finite subcover $U_{y_{1}}, \ldots, U_{y_{n}}$. Let $r=\min \left\{1 /\left\|T f_{y_{k}}\right\|: 1 \leq k \leq n\right\}$. For any $z \in Y, z \in U_{y_{k}}$ for some $k \leq n$, and $d(x, z) \geq\left|f_{y_{k}}(x)-f_{y_{k}}(z)\right| /\left\|T f_{y_{k}}\right\|>1 /\left\|T f_{y_{k}}\right\| \geq r$. Therefore $B(x, r) \subseteq U$ as desired.

(b) Let $\left(x_{n}\right)_{n=1}^{\infty}$ be any Cauchy sequence in $(X, d)$. Since $(X, \mathcal{T})$ is compact, this sequence has a convergent subsequence in $\mathcal{T}$, say $\left(x_{n_{k}}\right)_{k=1}^{\infty}$, such that $x_{n_{k}} \rightarrow x_{0}$ in $\mathcal{T}$ as $k \rightarrow \infty$. We want to show that $\lim _{n \rightarrow \infty} d\left(x_{n}, x_{0}\right)=0$. Note that since $\left(x_{n}\right)_{n=1}^{\infty}$ is Cauchy, if the limit of positive numbers $d\left(x_{n}, x_{0}\right)$ is 0 for any subsequence, it is 0 for the whole sequence. Thus, if this limit does not exist, it means that it has no subsequence which converges to 0 , and so there exists $r>0$ such that $d\left(x_{n}, x_{0}\right)>r$ for all $n \in \mathbb{N}$.

We are now going to show that $d\left(x_{n_{k}}, x_{0}\right) \rightarrow 0$ as $k \rightarrow \infty$. Take any $\varepsilon>0$ such that $\varepsilon<r$, and let $K \in \mathbb{N}$ be such that $k, l \geq K$ implies $d\left(x_{n_{k}}, x_{n_{l}}\right)<\varepsilon$. Fix any $k \geq K$. Since $d\left(x_{n_{k}}, x_{0}\right)>r$, there exists a function $f \in \operatorname{Dom}(T)$ such that $\|T f\|=1, f\left(x_{0}\right)=0$, and $\left|f\left(x_{n_{k}}\right)\right|>r$. Let $\delta>0$ be such that $\delta<r-\varepsilon$. Since $x_{n_{l}} \rightarrow x_{0}$ in $\mathcal{T}$, and $f$ is continuous in $\mathcal{T}$, there exists $M \geq K$ such that $l \geq M$ implies $\left|f\left(x_{n_{l}}\right)\right|<\delta$. But then for $l \geq M$

$$
\left|f\left(x_{n_{k}}\right)-f\left(x_{n_{l}}\right)\right|>|| f\left(x_{n_{k}}\right)|-| f\left(x_{n_{l}}\right)||>r-\delta>\varepsilon,
$$

which contradicts our assumption that $\left(x_{n_{k}}\right)_{k=1}^{\infty}$ is Cauchy. Thus $d\left(x_{n_{k}}, x_{0}\right) \rightarrow 0$ as $k \rightarrow \infty$.

Now, since $\left(x_{n}\right)_{n=1}^{\infty}$ is Cauchy, and it has a subsequence $\left(x_{n_{k}}\right)_{k=1}^{\infty}$ for which $d\left(x_{n_{k}}, x_{0}\right) \rightarrow 0$ as $k \rightarrow \infty$, we have that $d\left(x_{n}, x_{0}\right) \rightarrow 0$ as $n \rightarrow \infty$ as well. We conclude that $(X, d)$ is complete. 
(c) This is immediate from (a) and (b).

Remark 3.2. However, $\mathcal{T}_{d}$ need not be locally compact, even if $(X, \mathcal{T})$ is compact Hausdorff, as the following example shows. Let $X=\left\{x=\left(x_{k}\right)_{k=1}^{\infty}\right.$ : $\left.\left|x_{k}\right| \leq 1\right\}$, let $\rho(x, y)=\left(\sum_{k=1}^{\infty}\left(1 / k^{2}\right)\left|x_{k}-y_{k}\right|^{2}\right)^{1 / 2}$, and let $d(x, y)=$ $\min \left\{2,\left(\sum_{k=1}^{\infty}\left|x_{k}-y_{k}\right|^{2}\right)^{1 / 2}\right\}$, for $x, y \in X$. Let $(X, \mathcal{T})=(X, \rho)$, which is compact Hausdorff. However, $(X, d)$ is not locally compact, since for example 0 does not have a compact neighborhood $\left(l^{2}(\mathbb{N})\right.$ is not locally compact, and $X$ contains a unit ball of $\left.l^{2}(\mathbb{N})\right)$. We have $\mathcal{T}_{\rho} \subseteq \mathcal{T}_{d}$. Let $\hat{X}_{\rho}=\{(x, y) \in X \times X: x \neq y\}$ with product topology from $(X, \rho)$. Let $D_{d}$ be the operator with $\operatorname{Dom}\left(D_{d}\right) \subseteq C(X, \rho)$, Range $\left(D_{d}\right) \subseteq C_{b}\left(\hat{X}_{\rho}\right)$, defined by $\left(D_{d} f\right)(x, y)=(f(y)-f(x)) / d(x, y)$. It is easy to see that $d_{D_{d}}=d$.

The other way around, if we let $(X, \mathcal{T})=(X, d)$, then $C_{b}(X) \supseteq C(X, \rho)$. Let $D_{\rho}$ be the operator with $\operatorname{Dom}\left(D_{\rho}\right) \subseteq C(X, \rho) \subseteq C_{b}(X, d)$, Range $\left(D_{\rho}\right)=$ $C_{b}\left(\hat{X}_{\rho}\right)$, defined by $\left(D_{\rho} f\right)(x, y)=(f(y)-f(x)) / \rho(x, y)$. It is easy to see that $d_{D_{\rho}}=\rho$. So, we have an example where $(X, \mathcal{T})$ is not locally compact, but the metric $\rho$ defined by an operator makes it into a compact space, and in particular $\mathcal{T}_{\rho}$ is strictly contained in $\mathcal{T}$. Compared to the discussion prior to Proposition 3.1, in this case $\overline{\operatorname{Dom}\left(D_{\rho}\right)} \neq C_{b}(X)$.

Remark 3.3. Suppose that $(X, \mathcal{T})$ is compact Hausdorff, and that $(X, d)$ is a metric space such that $\mathcal{T}_{d}$ is finer then $\mathcal{T}$. It is not always true that there exists an operator $T$ such that $d=d_{T}$. Let $(X, \mathcal{T})=[0,1]$ with the usual topology, and let $(X, \rho)=\{-1\} \cup(0,1]$ with the topology inherited from $\mathbb{R}$, and the identification $\phi:(X, \rho) \rightarrow(X, \mathcal{T})$ given by $\phi(-1)=0$ and $\phi(x)=x$ if $x \in(0,1]$. Since $(X, \rho)$ is not complete, there is no $T$ for which $\rho=d_{T}$.

However, $\left(X, \mathcal{T}_{\rho}\right)$ is homeomorphic to $(X, d)=\{0\} \cup[1, \infty)$ with the identification given by $\psi:(X, \rho) \rightarrow(X, d)$ defined by $\psi(-1)=0$ and $\psi(x)=$ $1 / x$ for $x \in(0,1]$. We have $F=\psi \circ \phi:(X, \mathcal{T}) \rightarrow(X, d)$ given by $F(0)=0$ and $F(x)=1 / x$ for $x \in(0,1]$. Let $\hat{X}=X \times X \backslash\{(x, x): x \in X\}$ with inherited product topology from $(X, \mathcal{T})$. Let $D_{d}: C(X, \mathcal{T}) \rightarrow C_{b}(\hat{X})$ be defined by $\left(D_{d} f\right)(x, y)=(f(y)-f(x)) / d(F(x), F(y))$. It easy to check that $D_{d}$ defines the metric $d$.

Proposition 3.4. Let $(X, \mathcal{T})$ be a compact Hausdorff space, and d a possibly infinite metric on the set $X$, defined by an operator. The following are equivalent:

(1) $\mathcal{T}_{d}=\mathcal{T}$, i.e. $d$ induces the same topology on $X$ as $\mathcal{T}$.

(2) If a sequence is convergent in $\mathcal{T}$ then it is convergent in $\mathcal{T}_{d}$.

(3) $d: X \times X \rightarrow \mathbb{R}^{+} \cup\{\infty\}$ is continuous.

Proof. (1) $\Leftrightarrow(2)$ This holds with the assumption that $\mathcal{T}_{d} \supseteq \mathcal{T}$, which is true by Proposition 3.1(a). 
$(1) \Rightarrow(3)$ This is standard for any metric, even without the assumption of compactness.

(3) $\Rightarrow(1)$ We will show that if $\mathcal{T}_{d} \neq \mathcal{T}$, then $d$ is not continuous. Since by Proposition 3.1 (a) $\mathcal{T}_{d} \supseteq \mathcal{T}$, the only way $\mathcal{T}_{d}$ can fail to be the same as $\mathcal{T}$ is not to be compact. But, by the Borel-Lebesgue Theorem, $\left(X, \mathcal{T}_{d}\right)$ is compact if and only if it is sequentially compact, i.e. if and only if every sequence has a convergent subsequence. Hence, if $\left(X, \mathcal{I}_{d}\right)$ is not compact, there is a sequence $\left\{x_{n}\right\}$ in $X$ which does not have a $\mathcal{T}_{d}$ convergent subsequence. However, there is a subsequence $\left\{x_{n_{i}}\right\}$ of $\left\{x_{n}\right\}$ such that $x_{n_{i}} \rightarrow x$ in $\mathcal{T}$. So we have a sequence $\left(x_{n_{i}}, x\right) \rightarrow(x, x)$ in $X \times X$ such that $d\left(x_{n_{i}}, x\right)$ does not tend to $d(x, x)=0$. Hence $d$ can not be continuous.

We note that compactness of $(X, \mathcal{T})$ is crucial in $(3) \Rightarrow(1)$ above. For, let $(X, \rho)$ and $(X, d)$ be as in Remark 3.2. If $(X, \mathcal{T})=(X, d)$, then $\rho$ is continuous. However, $\mathcal{T}_{\rho} \neq \mathcal{T}$, and in fact $\mathcal{T}_{\rho}$ is strictly contained in $\mathcal{T}$.

The following observation is basic, but important for what follows.

Proposition 3.5. Let $(X, \mathcal{T})$ be a topological space, and let $B$ be a normed space. Let $T$ be a linear operator with $\operatorname{Dom}(T) \subseteq C_{b}(X)$, Range $(T) \subseteq B$, and $\operatorname{ker} T=\mathbb{C} 1$, which defines (possibly infinite) metric $d_{T}$ on the set $X$. Suppose that $\operatorname{Dom}(T)$ is dense in $C_{b}(X)$. If $T$ is bounded, then for all different $x, y \in X, d_{T}(x, y) \geq 2 /\|T\|$. Consequently, if $(X, \mathcal{T})$ is compact Hausdorff and $X$ is infinite, then $\mathcal{T}_{d_{T}} \neq \mathcal{T}$.

Proof. Note that $\{f \in \operatorname{Dom}(T):\|T f\| \leq 1\} \supseteq\{f \in \operatorname{Dom}(T):\|f\| \leq$ $1 /\|T\|\}$, since $\|f\| \leq 1 /\|T\|$ implies that $\|T f\| \leq\|T\|\|f\| \leq 1$. Therefore

$$
\begin{aligned}
d_{T}(x, y) & =\sup \{|f(x)-f(y)|: f \in \operatorname{Dom}(T) \&\|T f\| \leq 1\} \\
& \geq \sup \{|f(x)-f(y)|: f \in \operatorname{Dom}(T) \&\|f\| \leq 1 /\|T\|\} .
\end{aligned}
$$

But there exists a continuous function $g$ of norm $1 /\|T\|$ such that $g(x)=$ $1 /\|T\|$ and $g(y)=-1 /\|T\|$. Since $\operatorname{Dom}(T)$ is dense in $C(X)$, for every $\varepsilon>0$ there exists a function $f \in \operatorname{Dom}(T)$ such that $\|f\| \leq 1 /\|T\|$ and $|f(x)-f(y)| \geq(2 /\|T\|)-\varepsilon$. Thus $d_{T}(x, y) \geq 2 /\|T\|$, and $\mathcal{T}_{d_{T}}$ is discrete.

If $X$ is infinite, and $\mathcal{T}_{d_{T}}$ is discrete, it clearly fails to be compact. Thus, if $(X, \mathcal{T})$ is compact Hausdorff with $X$ infinite, and $T$ is bounded, then $\mathcal{T}_{d_{T}} \neq \mathcal{T}$.

This suggests that $T$ should not be bounded on any "large" subspace of $\operatorname{Dom}(T)$. We will explore that further.

\section{Lipschitz algebras and de Leeuw derivations.}

The relationship between compact metric spaces and Lipschitz algebras is deep, in the same way that the relationship between compact Hausdorff spaces and unital commutative $C^{*}$-algebras is. For example, their respective categories are (contravariantly) equivalent $([\mathbf{9}])$. Moreover, since any 
Lipschitz algebra is a domain of a specific operator - the de Leeuw derivation (see what follows), it is not surprising that any operator which defines a metric on a space is closely related to some de Leeuw derivation, the de Leeuw derivation associated to it. We now provide certain results about Lipschitz algebras and de Leeuw derivations (Theorem 4.7). These will be used to derive some necessary and sufficient conditions which $T$ should satisfy so that $\mathcal{T}_{d_{T}}=\mathcal{T}$ (Theorem 5.3). Basic facts about Lipschitz spaces and algebras can be found in $[\mathbf{1}],[\mathbf{4}],[\mathbf{5}],[\mathbf{1 2}]$, and [8]. We give definitions first.

Definition 4.1. A map $F: X \rightarrow Y$ from a metric space $\left(X, d_{X}\right)$ to a metric space $\left(Y, d_{Y}\right)$ is said to be a Lipschitz map if there exists a constant $M$ such that for all $x, y$ in $X$

$$
d_{Y}(F(x), F(y)) \leq M d_{X}(x, y) .
$$

The smallest such constant is called the Lipschitz constant of $F$. We denote it by $\mathrm{p}(F)$.

We can write $\mathrm{p}(F)$ explicitly as

$$
\begin{aligned}
\mathrm{p}(F) & =\inf \left\{M \in \mathbb{R}^{+}: d_{Y}(F(x), F(y)) \leq M d_{X}(x, y) \forall x, y \in X\right\} \\
& =\sup \left\{d_{Y}(F(x), F(y)) / d_{X}(x, y): x, y \in X \& x \neq y\right\} .
\end{aligned}
$$

When $Y$ is a normed space $\mathrm{p}(F)$ is also called the Lipschitz norm of $F$ (it is in fact a semi-norm). Thus, $F$ is Lipschitz if $\mathrm{p}(F)<\infty$.

Let $\left(X, d_{X}\right)$ be a metric space. $\operatorname{Lip}\left(X, d_{X}\right)$ will denote the set of all bounded complex valued continuous functions on $\left(X, d_{X}\right)$ which are Lipschitz with respect to $d_{X}$ and the standard metric on $\mathbb{C}$. Let $\|f\|_{\infty}=$ $\sup \{|f(x)|: x \in X\}$. Define a norm on $\operatorname{Lip}\left(X, d_{X}\right)$ by $\|f\|=\|f\|_{\infty}+\mathrm{p}(f)$. With respect to pointwise operations $\operatorname{Lip}\left(X, d_{X}\right)$ is a self-adjoint Banach *-algebra over $X\left(\left\|a^{*}\right\|=\|a\|, a \in\right.$ the algebra $)$.

Definition 4.2. A commutative Banach $*$-algebra $A$ is called Lipschitz if there exists a metric space $\left(X, d_{X}\right)$ so that $A=\operatorname{Lip}\left(X, d_{X}\right)$.

If $\left(X, d_{X}\right)$ is compact, then $\operatorname{Lip}\left(X, d_{X}\right)=\{f \in C(X): \mathrm{p}(f)<\infty\}$, and it is a unital, natural, regular, self-adjoint Banach function algebra over $X$ (see $[\mathbf{1 2}])$.

So far we have been concerned with obtaining metrics from operators. We now show that given any metric space $(X, d)$, there exists an operator, in fact a derivation $D$ with $\operatorname{Dom}(D) \subseteq C(X)$ which defines $d$. This derivation has been known for a long time and used by a number of authors. It appeared for the first time in [4], which is the reason why we will call it the de Leeuw derivation. It is used in [1] and also disguised in [11] (see Remark 4.8). It turns out that this derivation is important in our considerations. We will show that it is in a sense maximal among the operators which define the same metric $d$. We will also show that when $(X, d)$ is compact, $D$ is unbounded on every infinite dimensional subspace of its domain. 
Proposition 4.3. Let $(X, d)$ be any metric space. Let $Y=\{(x, y) \in X \times$ $X: x \neq y\}$, and let $B=C_{b}(Y)$. For $f \in C_{b}(X)$ and $b \in B$ let $f . b$ and $b . f$ be defined by $(f . b)(x, y)=f(x) b(x, y)$ and $(b . f)(x, y)=f(y) b(x, y),(x, y) \in Y$. Let $D: C_{b}(X) \rightarrow B$ be defined by

$$
(D f)(x, y)=\frac{f(y)-f(x)}{d(x, y)}
$$

for $(x, y) \in Y$. Then $B$ is a Banach $C_{b}(X)$-bimodule, $D$ is a derivation with $\operatorname{Dom}(D)=\operatorname{Lip}(X, d)=\left\{f \in C_{b}(X):\|D f\|<\infty\right\}$, and $D$ is a closed operator.

Furthermore, if $d_{D}$ denotes the metric defined by $D$, then $d_{D}=d$.

Proof. Since $\|D f\|=\mathrm{p}(f)$, it is clear that $\operatorname{Dom}(D)=\operatorname{Lip}(X, d)$. It is obvious that $D$ is linear and that $D\left(1_{X}\right)=0$. Let $f, g \in \operatorname{Dom}(D)$. Then

$$
\begin{aligned}
(D(f g))(x, y) & =\frac{f(y) g(y)-f(x) g(x)}{d(x, y)} \\
& =\frac{f(y) g(y)-f(x) g(y)+f(x) g(y)-f(x) g(x)}{d(x, y)} \\
& =(D f . g)(x, y)+(f . D g)(x, y)=(D f . g+f . D g)(x, y) .
\end{aligned}
$$

Thus, $D$ is a derivation.

It is true for any operator $D: A \rightarrow B(A, B$ Banach spaces), that $\operatorname{Dom}(T)$ is a Banach space under the norm $\|f\|+\|D f\|$ if and only if $D$ is closed. Since we know that $\operatorname{Lip}(X, d)$ is a Banach space, $D$ is closed. It is not difficult to check that directly.

To show that $d_{D}=d$, let $x, y \in X$. By definition,

$$
\begin{aligned}
d_{D}(x, y) & =\sup \{|f(x)-f(y)|: f \in \operatorname{Dom}(D) \&\|D f\| \leq 1\} \\
& =\sup \{|f(x)-f(y)|: f \in \operatorname{Lip}(X, d) \& \mathrm{p}(f) \leq 1\} .
\end{aligned}
$$

So for any $\varepsilon>0$ there exists $f \in \operatorname{Lip}(X, d)$ with $\mathrm{p}(f) \leq 1$ and $|f(x)-f(y)|>$ $d_{D}(x, y)-\varepsilon$. But $|f(x)-f(y)| \leq \mathrm{p}(f) d(x, y) \leq d(x, y)$. Thus, for any $\varepsilon>0, d(x, y)>d_{D}(x, y)-\varepsilon$, and consequently $d(x, y) \geq d_{D}(x, y)$. On the other hand, for $x, y \in X$, the function $f$ defined by $f(s)=d(s, y)$ if $d(s, y) \leq d(x, y)$ and $f(s)=d(x, y)$ if $d(s, y) \geq d(x, y)$, for $s \in X$, is bounded and Lipschitz with $\mathrm{p}(f)=1$. So $d_{D}(x, y) \geq|f(x)-f(y)|=d(x, y)$. Hence $d_{D}(x, y)=d(x, y)$ for any $x, y \in X$.

We say that such a derivation $D$ is the de Leeuw derivation defined by metric $d$.

We now prove a partial converse to Proposition 3.5.

Proposition 4.4. Let $(X, d)$ be a metric space. If there exists a constant $r>0$ such that $x \neq y$ implies that $d(x, y) \geq r$ (i.e. $(X, d)$ is uniformly discrete), then $D_{d}$ is bounded, and $\left\|D_{d}\right\| \leq 2 / r$. 
Proof. Let $f \in \operatorname{Dom}\left(D_{d}\right)$. Let $\varepsilon>0$, and $x, y \in X$ be such that $\left\|D_{d} f\right\|=$ $\mathrm{p}(f)<(|f(x)-f(y)| / d(x, y))+\varepsilon\|f\|$. Since $|f(x)-f(y)| \leq 2\|f\|$ we have $\left\|D_{d} f\right\|<(2\|f\| / d(x, y))+\varepsilon\|f\|$, i.e. $\left\|D_{d} f\right\| /\|f\|<(2 / d(x, y))+\varepsilon \leq$ $(2 / r)+\varepsilon$. Since this holds for any $\varepsilon>0$, we have $\left\|D_{d} f\right\| /\|f\| \leq 2 / r$. Hence $D_{d}$ is bounded, and $\left\|D_{d}\right\| \leq 2 / r$.

The following proposition shows how the de Leeuw derivation is related to other operators which define the same metric $d$.

Proposition 4.5. Let $(X, \mathcal{T})$ be a topological space, and let $B$ be a normed space. Let $T$ be a linear operator with $\operatorname{Dom}(T) \subseteq C_{b}(X)$, $\operatorname{ker} T=\mathbb{C} 1$, and Range $(T) \subseteq B$, which defines the metric $d$ on the set $X$. Let $\hat{D}$ be the derivation defined by $d$, as in Proposition 4.3, mapping into the module $\hat{B}$. Then $\operatorname{Dom}(T)$ is a subspace of $\operatorname{Lip}(X, d)=\operatorname{Dom}(\hat{D})$, and for $g \in \operatorname{Dom}(T)$, $\|T g\| \geq\|\hat{D} g\|$. There exists a linear one-to-one contraction $\imath: \operatorname{Range}(T) \rightarrow$ Range $(\hat{D})$. Furthermore, if $D=\left.\hat{D}\right|_{\operatorname{Dom}(T)}$, then $D$ defines the same metric $d$ as $T$ does.

Proof. Let $g \in \operatorname{Dom}(T)$. Since $d(x, y)=\sup \{|f(x)-f(y)|:\|T f\| \leq 1\}$, $|g(x)-g(y)| \leq d(x, y)\|T g\|$ for any $x, y \in X$. So $g \in \operatorname{Dom}(\hat{D})$ and $\|\hat{D} g\|=$ $\mathrm{p}(g)=\sup \{|g(x)-g(y)| / d(x, y): x, y \in X \& x \neq y\} \leq\|T g\|$. This proves the first assertion, that if $g \in \operatorname{Dom}(T)$, then $g \in \operatorname{Dom}(\hat{D})=\operatorname{Lip}(X, d)$, and $\|T g\| \geq\|\hat{D} g\|$. The existence of a linear one-to-one contraction $\imath: \operatorname{Range}(T)$ $\rightarrow$ Range $(\hat{D})$ follows directly from the previous assertion, and the fact that $\operatorname{ker} T=\operatorname{ker} \hat{D}=\mathbb{C} 1$.

To prove the last assertion, that $d_{D}=d$, fix any $x, y \in X$, and let $\varepsilon>0$. There exists $f \in \operatorname{Dom}(T)$ such that $|f(x)-f(y)| /\|T f\|>d(x, y)-\varepsilon$. So, since $\|D f\|=\|\hat{D} f\|$,

$$
\begin{aligned}
d(x, y) & \geq \frac{|f(x)-f(y)|}{\|D f\|}=\frac{|f(x)-f(y)|}{\|T f\|} \frac{\|T f\|}{\|D f\|} \\
& \geq \frac{|f(x)-f(y)|}{\|T f\|}>d(x, y)-\varepsilon .
\end{aligned}
$$

Since this is true for any $\varepsilon$, we obtain that

$$
d_{D}(x, y)=\sup \{|f(x)-f(y)| /\|D f\|: f \in \operatorname{Dom}(T)\}=d(x, y),
$$

as desired.

The derivation $\hat{D}=D_{d_{T}}$ in the previous proposition we will call the de Leeuw derivation associated to the operator $T$. Note that $\operatorname{Lip}(X, d)=$ $\operatorname{Dom}(\hat{D})$ is not necessarily contained in $C_{b}(X)$, since it might be that $\mathcal{T}_{d} \neq \mathcal{T}$. Thus $\hat{D}$ is not an operator from $C_{b}(X)$. However, $D$ is.

Proposition 4.5 suggests that there is a partial order relation on $\mathcal{D}(X, d)$, the family of all operators on $C_{b}(X)$ which define metric $d$. Consider a partial 
order relation on $\mathcal{D}(X, d), \prec$, defined by $D_{1} \prec D_{2}$ if $\operatorname{Dom}\left(D_{1}\right) \subseteq \operatorname{Dom}\left(D_{2}\right)$ and $\forall x \in \operatorname{Dom}\left(D_{1}\right),\left\|D_{1} x\right\| \geq\left\|D_{2} x\right\|$. It induces an equivalence relation on $\mathcal{D}(X, d), \approx$, by $D_{1} \approx D_{2}$ if $\operatorname{Dom}\left(D_{1}\right)=\operatorname{Dom}\left(D_{2}\right)$ and $\forall x \in \operatorname{Dom}\left(D_{1}\right)$, $\left\|D_{1} x\right\|=\left\|D_{2} x\right\|$. By Proposition 4.5, there exists a unique (up to the above equivalence) maximal element in $\mathcal{D}(X, d)$, namely the derivation defined by $d$, as in Proposition 4.3.

We quote a theorem from [5], which will be used to show that if $D$ is a de Leeuw derivation defined by a metric, then it is unbounded on every infinite dimensional subspace of its domain.

Theorem 4.6. (J. Johnson, [5], Theorem 3.5.) Let $(X, d)$ be any metric space. Then the following are equivalent:

(a) $(X, d)$ is precompact.

(b) The unit ball of $\operatorname{Lip}\left(X, d^{\alpha}\right), 0<\alpha \leq 1$, is compact for the sup-norm topology.

(c) The unit ball of $\operatorname{Lip}\left(X, d^{\beta}\right)$ is compact in $\operatorname{lip}\left(X, d^{\alpha}\right)$ for $0<\alpha<\beta \leq 1$.

Here $\operatorname{lip}\left(X, d^{\alpha}\right)=\left\{f \in \operatorname{Lip}\left(X, d^{\alpha}\right):|f(x)-f(y)| / d(x, y)^{\alpha} \rightarrow 0\right.$ as $d(x, y) \rightarrow 0\}$. Recall that a metric space is precompact if its completion is compact. Note that the norm on $\operatorname{Lip}(X, d)$ used here is $\max \left\{\|f\|_{\infty}, \mathrm{p}(f)\right\}$, but it is equivalent to the one we use. The main argument in one direction of the proof of the above theorem is the Arzela-Ascoli theorem. We will do a generalization of this theorem later (Theorem 5.1), but now we add some more equivalent statements to it. We follow the common practice in Lipschitz algebras in using the following notation: $\mathrm{p}_{\alpha}(f)=$ $\sup \left\{|f(x)-f(y)| / d(x, y)^{\alpha}: x \neq y\right\}$, for $0<\alpha \leq 1$.

Theorem 4.7. Let $(X, d)$ be any metric space, and let $D_{d}$ be the de Leeuw derivation defined by $d$. The following are equivalent:

(a) The space $(X, d)$ is precompact.

(d) For $0<\alpha \leq 1$, the derivation $D_{d^{\alpha}}$ is unbounded on every infinite dimensional subspace of its domain. Equivalently, the function $F$ : $\operatorname{Lip}\left(X, d^{\alpha}\right) \backslash\{0\} \rightarrow \mathbb{R}^{+}$defined by $F(f)=\mathrm{p}_{\alpha}(f) /\|f\|_{\infty}$, is unbounded on every infinite dimensional subspace of $\operatorname{Lip}\left(X, d^{\alpha}\right)$.

(e) For $0<\alpha<\beta \leq 1, D_{d^{\beta}}$ viewed as a derivation from $\operatorname{Lip}\left(X, d^{\alpha}\right)$, is unbounded on every infinite dimensional subspace of its domain $\operatorname{Lip}\left(X, d^{\beta}\right)$. Equivalently, the function $G: \operatorname{Lip}\left(X, d^{\beta}\right) \backslash \mathbb{C} 1 \rightarrow \mathbb{R}^{+}$defined by $G(f)=$ $\mathrm{p}_{\beta}(f) /\left(\|f\|_{\infty}+\mathrm{p}_{\alpha}(f)\right)$ is unbounded on every infinite dimensional subspace of its domain.

Proof. Recall that for $0<\alpha<\beta \leq 1, C_{b}(X) \supseteq \operatorname{Lip}\left(X, d^{\alpha}\right) \supseteq \operatorname{Lip}\left(X, d^{\beta}\right)$. Then $\hat{B}=C_{b}(\hat{X})$, where $\hat{X}=\{(x, y) \in X \times X: x \neq y\}$, is a Banach $\operatorname{Lip}\left(X, d^{\alpha}\right)$-module, as well as a Banach $C_{b}(X)$-module. The module operations are the same as for $C_{b}(X)$ : For $f \in A=\operatorname{Lip}\left(X, d^{\alpha}\right)$ (or $\left.C_{b}(X)\right)$ and $b \in \hat{B},(f . b)(x, y)=f(x) b(x, y)$ and $(b . f)(x, y)=f(y) b(x, y)$. Thus the de 
Leeuw derivation $D_{d^{\beta}}\left(D_{d^{\beta}}(x, y)=(f(y)-f(x)) / d^{\beta}(x, y)\right)$ whose domain is $\operatorname{Lip}\left(X, d^{\beta}\right)$ can be thought of as an unbounded derivation either from $C_{b}(X)$ (as we did so far), or it can be thought of as an unbounded derivation from $\operatorname{Lip}\left(X, d^{\alpha}\right)(0<\alpha<\beta \leq 1)$, which is done here in part (e).

For $(\mathrm{a}) \Leftrightarrow(\mathrm{d})$, it is enough to consider $\alpha=1$, since $d^{\alpha}$ is topologically equivalent to $d$, and they share the same Cauchy sequences.

$(a) \Rightarrow(d)$. Suppose that to the contrary, there exists an infinite dimensional subspace $V$ of $\operatorname{Lip}(X, d)$ on which $F$ is bounded, say $\sup \{F(f): f \in$ $V\}=P(V)$. First, we show that $F$ is a continuous function on $\operatorname{Lip}(X, d) \backslash$ $\{0\}$. Let $f \in \operatorname{Lip}(X, d) \backslash\{0\}, \varepsilon>0, \delta=\|f\|_{\infty} \min \{(1 / 4), \varepsilon / 4(1+F(f))\}$, and let $g \in \operatorname{Lip}(X, d) \backslash\{0\}$ be such that $\|g-f\|=\|g-f\|_{\infty}+\mathrm{p}(g-f)<\delta$. Then $\|g\|_{\infty} \geq\|f\|_{\infty}-\|g-f\|_{\infty}>\|f\|_{\infty}-\delta>\|f\|_{\infty}-\|f\|_{\infty} / 2=\|f\|_{\infty} / 2$, i.e. $\|f\|_{\infty} /\|g\|_{\infty}<2$. We have

$$
\begin{aligned}
|F(g)-F(f)| & =\left|\frac{\mathrm{p}(g)}{\|g\|_{\infty}}-\frac{\mathrm{p}(f)}{\|f\|_{\infty}}\right| \\
& \leq \frac{|\mathrm{p}(g)-\mathrm{p}(f)|}{\|g\|_{\infty}}+\frac{\mathrm{p}(f)}{\|f\|_{\infty}} \frac{\left|\|f\|_{\infty}-\|g\|_{\infty}\right|}{\|g\|_{\infty}} \\
& \leq \frac{\mathrm{p}(g-f)}{\|g\|_{\infty}}+F(f) \frac{\|f-g\|_{\infty}}{\|g\|_{\infty}}<\frac{\delta}{\|g\|_{\infty}}+F(f) \frac{\delta}{\|g\|_{\infty}} \\
& =\delta \frac{(1+F(f))}{\|g\|_{\infty}} \leq \frac{\varepsilon\|f\|_{\infty}}{4(1+F(f))} \frac{(1+F(f))}{\|g\|_{\infty}}=\frac{\varepsilon\|f\|_{\infty}}{4\|g\|_{\infty}}<\varepsilon,
\end{aligned}
$$

by the choice of $\delta$. Thus, $F$ is continuous on $\operatorname{Lip}(X, d) \backslash\{0\}$. Since on $V$ it is also bounded, it is bounded on $\bar{V}$ as well (the closure is taken in $\operatorname{Lip}(X, d)$ ), and with the same bound, $P(V)$. On $\bar{V}$ we have that

$$
\|f\|_{\infty} \leq\|f\|_{\infty}+\mathrm{p}(f) \leq(1+P(V))\|f\|_{\infty},
$$

i.e. the Lipschitz and the supremum norms are equivalent. That means that $\bar{V}$ is closed also as a subspace of $C(X)$.

Let $B=\left\{f \in \bar{V}:\|f\|_{\infty}<1 /(1+P(V))\right\}$, and $\bar{B}=\left\{f \in \bar{V}:\|f\|_{\infty} \leq\right.$ $1 /(1+P(V))\}$. Then $B$ is a neighborhood of the origin in $\bar{V}$, and $\bar{B}$ is a closed subset (in supremum norm) of the unit ball $B_{1}$ of $\operatorname{Lip}(X, d)$. But, by the quoted Theorem 4.6, $B_{1}$ is compact in supremum norm, and so is $\bar{B}$. That means that $\bar{V}$ is locally compact (in supremum norm). But then, it is finite dimensional, since every locally compact topological vector space is finite dimensional. So, $\bar{V}$ and thus $V$ are finite dimensional, which is a contradiction to our assumption. Hence $D_{d}$ has to be unbounded on $V$.

$(\mathrm{d}) \Rightarrow(\mathrm{a})$. Suppose that $(X, d)$ is not precompact. Then it is not totally bounded, and so there is a sequence $\left(x_{n}\right)_{n=1}^{\infty}$, and $r>0$ such that $d\left(x_{m}, x_{n}\right)>2 r$ for all $m, n \in \mathbb{N}$. Let $\left(f_{j}\right)_{j=1}^{\infty}$ be a sequence of functions defined by $f_{j}(x)=\max \left\{0, r-d\left(x, x_{j}\right)\right\}$. Then $\left\|D_{d} f_{j}\right\|=1,\left\|f_{j}\right\|_{\infty}=r$, and the functions $f_{j}$ have pairwise nonintersecting supports. Let $V=$ 
$\operatorname{span}\left(f_{j}: j \in \mathbb{N}\right)$. We show that $D_{d}$ is bounded on $V$. If $f \in V$, then $f=\sum_{i=1}^{l} a_{i} f_{i}$. Clearly, $\|f\|_{\infty}=r \max \left\{\left|a_{i}\right|\right\}$. Suppose that $\|f\|_{\infty}=r$, and compute $\left\|D_{d} f\right\|=\sup \{|f(x)-f(y)| / d(x, y): x \neq y\}$. We have that

$$
f(x)-f(y)=a_{m} f_{m}(x)-a_{n} f_{n}(y)=a_{m}\left(r-d\left(x, x_{m}\right)\right)-a_{n}\left(r-d\left(y, x_{n}\right)\right)
$$

for some $m, n \leq l$. For any fixed $R=d(x, y)$, the largest possible $\sup \{|f(x)-f(y)| / d(x, y): x \neq y, d(x, y)=R\}$ is achieved when $\left|a_{m}\right|=1$ and $a_{n}=-a_{m}$, and for these

$$
\begin{aligned}
\frac{|f(x)-f(y)|}{d(x, y)} & =\frac{r-d\left(x, x_{m}\right)+r-d\left(y, x_{n}\right)}{d(x, y)} \\
& \leq \frac{d\left(x_{m}, x_{n}\right)-d\left(x, x_{m}\right)-d\left(y, x_{n}\right)}{d(x, y)} \leq 1
\end{aligned}
$$

Thus $\sup \left\{\left\|D_{d} f\right\| /\|f\|_{\infty}: f \in V=\operatorname{span}\left(f_{n}\right)\right\}=1 / r<\infty$. Boundedness of $D_{d}$ on the infinite dimensional subspace $V$ contradicts our assumption, and therefore $(X, d)$ must be precompact.

(a) $\Rightarrow(\mathrm{e})$. Suppose that to the contrary, there exists an infinite dimensional subspace $V$ of $\operatorname{Lip}\left(X, d^{\beta}\right)$ on which $G$ is bounded, say $\sup \{G(f)$ : $f \in V\}=Q(V)$. First, we show that the function $H: \operatorname{Lip}\left(X, d^{\beta}\right) \backslash$ $\{f: f(x)=c\}$ defined by $H(f)=\mathrm{p}_{\beta}(f) / \mathrm{p}_{\alpha}(f)$ is a continuous function on its domain. Since $X$ is precompact, $d$ is bounded on $X$. Let $\operatorname{Diam}(X, d)=\sup \{d(x, y): x, y \in X\}$, and let $d_{X}=\max \{1, \operatorname{Diam}(X, d)\}$. For any $f \in \operatorname{Lip}\left(X, d^{\beta}\right)$,

$$
\begin{aligned}
\mathrm{p}_{\alpha}(f) & =\sup \left\{\frac{|f(x)-f(y)|}{d(x, y)^{\alpha}}: x, y \in X \& x \neq y\right\} \\
& =\sup \left\{\frac{|f(x)-f(y)|}{d(x, y)^{\beta}} d(x, y)^{\beta-\alpha}: x, y \in X \& x \neq y\right\} \\
& \leq \sup \left\{\frac{|f(x)-f(y)|}{d(x, y)^{\beta}}: x, y \in X \& x \neq y\right\} d_{X}^{\beta-\alpha}=\mathrm{p}_{\beta}(f) d_{X}^{\beta-\alpha},
\end{aligned}
$$

that is, $\mathrm{p}_{\alpha}(f) / \mathrm{p}_{\beta}(f) \leq d_{X}^{\beta-\alpha}$. Let $f \in \operatorname{Lip}\left(X, d^{\beta}\right), \quad \varepsilon>0$, $\delta=\mathrm{p}_{\alpha}(f) \min \left\{(1 / 4), \varepsilon d_{X}^{\beta-\alpha} / 4\left(1+d_{X}^{\beta-\alpha} H(f)\right)\right\}$, and let $g \in \operatorname{Lip}\left(X, d^{\beta}\right)$ be such that $\|g-f\|_{\beta}=\|g-f\|_{\infty}+\mathrm{p}_{\beta}(g-f)<\delta$. Then $\mathrm{p}_{\alpha}(g) \geq \mathrm{p}_{\alpha}(f)-$ $\mathrm{p}_{\alpha}(g-f)>\mathrm{p}_{\alpha}(f)-\delta>\mathrm{p}_{\alpha}(f)-\left(\mathrm{p}_{\alpha}(f) / 4\right)>\left(\mathrm{p}_{\alpha}(f) / 2\right)$, so that $\left(1 / \mathrm{p}_{\alpha}(g)\right)<$ 
$\left(2 / \mathrm{p}_{\alpha}(f)\right)$. We have

$$
\begin{aligned}
& |H(g)-H(f)| \\
& =\left|\frac{\mathrm{p}_{\beta}(g)}{\mathrm{p}_{\alpha}(g)}-\frac{\mathrm{p}_{\beta}(f)}{\mathrm{p}_{\alpha}(f)}\right|=\left|\frac{\mathrm{p}_{\beta}(g)}{\mathrm{p}_{\alpha}(g)}-\frac{\mathrm{p}_{\beta}(f)}{\mathrm{p}_{\alpha}(g)}+\frac{\mathrm{p}_{\beta}(f)}{\mathrm{p}_{\alpha}(g)}-\frac{\mathrm{p}_{\beta}(f)}{\mathrm{p}_{\alpha}(f)}\right| \\
& \leq \frac{\left|\mathrm{p}_{\beta}(g)-\mathrm{p}_{\beta}(f)\right|}{\mathrm{p}_{\alpha}(g)}+\mathrm{p}_{\beta}(f)\left|\frac{1}{\mathrm{p}_{\alpha}(g)}-\frac{1}{\mathrm{p}_{\alpha}(f)}\right| \\
& =\frac{\left|\mathrm{p}_{\beta}(g)-\mathrm{p}_{\beta}(f)\right|}{\mathrm{p}_{\alpha}(g)}+\frac{\mathrm{p}_{\beta}(f)}{\mathrm{p}_{\alpha}(f)} \frac{\left|\mathrm{p}_{\alpha}(f)-\mathrm{p}_{\alpha}(g)\right|}{\mathrm{p}_{\alpha}(g)} \\
& \leq \frac{\mathrm{p}_{\beta}(g-f)}{\mathrm{p}_{\alpha}(g)}+H(f) \frac{\mathrm{p}_{\alpha}(g-f)}{\mathrm{p}_{\alpha}(g)} \leq \frac{\mathrm{p}_{\beta}(g-f)}{\mathrm{p}_{\alpha}(g)}+H(f) \frac{d_{X}^{\beta-\alpha} \mathrm{p}_{\beta}(g-f)}{\mathrm{p}_{\alpha}(g)} \\
& =\frac{\mathrm{p}_{\beta}(g-f)}{\mathrm{p}_{\alpha}(g)}\left(1+d_{X}^{\beta-\alpha} H(f)\right)<\delta \frac{2}{\mathrm{p}_{\alpha}(f)}\left(1+d_{X}^{\beta-\alpha} H(f)\right) \\
& \leq \frac{\varepsilon \mathrm{p}_{\alpha}(f)}{4\left(1+d_{X}^{\beta-\alpha} H(f)\right)} \frac{2\left(1+d_{X}^{\beta-\alpha} H(f)\right)}{\mathrm{p}_{\alpha}(f)}<\varepsilon,
\end{aligned}
$$

by the choice of $\delta$. Thus, $H$ is continuous on $\operatorname{Lip}\left(X, d^{\beta}\right) \backslash\{f: f(x)=c\}$. From $(\mathrm{a}) \Rightarrow(\mathrm{d})$ we know that $F(f)=\mathrm{p}_{\beta}(f) /\|f\|_{\infty}$ is also continuous on $\operatorname{Lip}\left(X, d^{\beta}\right) \backslash\{0\}$. Hence $G(f)=\mathrm{p}_{\beta}(f) /\left(\|f\|_{\infty}+\mathrm{p}_{\alpha}(f)\right)=1 /((1 / F(f))+$ $(1 / G(f)))$ is continuous on $\operatorname{Lip}\left(X, d^{\beta}\right) \backslash\{f: f(x)=c\}$. Since on $V$ it is also bounded, it is bounded on $\bar{V}$ as well (the closure is taken in $\operatorname{Lip}\left(X, d^{\beta}\right)$ ), and with the same bound, $Q(V)$. On $\bar{V}$ we have that

$$
\begin{aligned}
\|f\|_{\alpha} & =\|f\|_{\infty}+\mathrm{p}_{\alpha}(f) \leq\|f\|_{\infty}+d_{X}^{\beta-\alpha} \mathrm{p}_{\beta}(f) \leq d_{X}^{\beta-\alpha}\left(\|f\|_{\infty}+\mathrm{p}_{\beta}(f)\right) \\
& =d_{X}^{\beta-\alpha}\|f\|_{\beta} \leq d_{X}^{\beta-\alpha}\left(\|f\|_{\infty}+Q(V)\left(\|f\|_{\infty}+\mathrm{p}_{\alpha}(f)\right)\right) \\
& \leq d_{X}^{\beta-\alpha}(1+Q(V))\left(\|f\|_{\infty}+\mathrm{p}_{\alpha}(f)\right)=d_{X}^{\beta-\alpha}(1+Q(V))\|f\|_{\alpha},
\end{aligned}
$$

i.e. the two norms are equivalent. That means that $\bar{V}$ is closed also as a subspace of $\operatorname{Lip}\left(X, d^{\alpha}\right)$.

Let $B=\left\{f \in \bar{V}:\|f\|_{\alpha}<1 /(1+Q(V))\right\}$, and $\bar{B}=\left\{f \in \bar{V}:\|f\|_{\alpha} \leq\right.$ $1 /(1+Q(V))\}$. Then $B$ is a neighborhood of the origin in $\bar{V}$, and $\bar{B}$ is a closed subset (in $\operatorname{Lip}\left(X, d^{\alpha}\right)$ norm) of the unit ball $B_{1}$ of $\operatorname{Lip}\left(X, d^{\alpha}\right)$. But, by the quoted Theorem 4.6, $B_{1}$ is compact in the $\operatorname{Lip}\left(X, d^{\alpha}\right)$ norm, and so is $\bar{B}$. That means that $\bar{V}$ is locally compact (in $\operatorname{Lip}\left(X, d^{\alpha}\right)$ norm). But then, it is finite dimensional. So, $\bar{V}$ and thus $V$ are finite dimensional, which is a contradiction to our assumption. Hence $D_{d^{\beta}}$ has to be unbounded on $V$.

$(\mathrm{e}) \Rightarrow(\mathrm{a})$. Suppose that $(X, d)$ is not precompact. Then it is not totally bounded, and so there is a sequence $\left(x_{n}\right)_{n=1}^{\infty}$, and $r>0$ such that $d\left(x_{m}, x_{n}\right)>2^{(1 / \beta)} r$ for all $m, n \in \mathbb{N}$. Let $\left(f_{j}\right)_{j=1}^{\infty}$ be a sequence of functions defined by $f_{j}(x)=\max \left\{0, r^{\beta}-d^{\beta}\left(x, x_{j}\right)\right\}$. Then $\left\|D_{d^{\beta}} f_{j}\right\|=1,\left\|f_{j}\right\|_{\infty}=r^{\beta}$, 
and the functions $f_{j}$ have pairwise nonintersecting supports. Let $V=$ $\operatorname{span}\left(f_{j}: j \in \mathbb{N}\right)$. We show that $D_{d^{\beta}}$ is bounded on $V$. If $f \in V$, then $f=\sum_{i=1}^{l} a_{i} f_{i}$. Clearly, $\|f\|_{\infty}=r^{\beta} \max \left\{\left|a_{i}\right|\right\}$. Suppose that $\|f\|_{\alpha}=r^{\beta}$, and compute $\left\|D_{d^{\beta}} f\right\|=\sup \left\{|f(x)-f(y)| / d^{\beta}(x, y): x \neq y\right\}$. We have that

$$
\begin{aligned}
f(x)-f(y) & =a_{m} f_{m}(x)-a_{n} f_{n}(y) \\
& =a_{m}\left(r^{\beta}-d^{\beta}\left(x, x_{m}\right)\right)-a_{n}\left(r^{\beta}-d^{\beta}\left(y, x_{n}\right)\right)
\end{aligned}
$$

for some $m, n \leq l$. For any fixed $R=d(x, y)$, the largest possible $\sup \left\{|f(x)-f(y)| / d^{\beta}(x, y): x \neq y, d(x, y)=R\right\}$ is achieved when $\left|a_{m}\right|=1$ and $a_{n}=-a_{m}$, and for these

$$
\begin{aligned}
\frac{|f(x)-f(y)|}{d^{\beta}(x, y)} & =\frac{r^{\beta}-d^{\beta}\left(x, x_{m}\right)+r^{\beta}-d^{\beta}\left(y, x_{n}\right)}{d^{\beta}(x, y)} \\
& \leq \frac{d^{\beta}\left(x_{m}, x_{n}\right)-d^{\beta}\left(x, x_{m}\right)-d^{\beta}\left(y, x_{n}\right)}{d^{\beta}(x, y)} \leq 1
\end{aligned}
$$

Thus $\sup \left\{\left\|D_{d^{\beta}} f\right\| /\|f\|_{\alpha}: f \in V=\operatorname{span}\left(f_{n}\right)\right\}=\left(1 / r^{\beta}\right)<\infty$. Boundedness of $D_{d}^{\beta}$ on the infinite dimensional subspace $V$ contradicts our assumption, and therefore $(X, d)$ must be precompact.

Remark 4.8. Finally, we remark that the de Leeuw derivation is not so far removed from the unbounded Fredholm module of Connes's definition as it might seem without careful inspection. Let $(X, d)$ be any metric space, and let $A=C_{b}(X)$. We present M. Rieffel's construction [11] of $(\mathcal{H}, D)$, and a $*$-representation $r$ of $A$ on $\mathcal{H}$ such that $T: A \rightarrow \mathcal{B}(\mathcal{H})$ defined by $T f=[D, r(f)]$ is equal to $M\left(D_{d}(f)\right) F$, where $D_{d}$ is the de Leeuw derivation, $M$ is the multiplication operator, and $F$ is the flip (see what follows for the details). Denote by $\hat{X}$ the set $\{(x, y) \in X \times X: x \neq y\}$, and by $\Delta$ the set $\{(x, x): x \in X\}$. Let $\mu$ be any measure on $\hat{X}$ such that $M: C_{b}(\hat{X}) \rightarrow$ $\mathcal{B}(\mathcal{H})$, where $\mathcal{H}=L^{2}(\hat{X}, \mu)$, defined by $(M(f) \xi)(x, y)=f(x, y) \xi(x, y)$, for $f \in C_{b}(\hat{X}), \xi \in \mathcal{H}$, and $(x, y) \in \hat{X}$ (multiplication operator by $f$ ), is an isometry. Let $L: C_{b}(X) \rightarrow C_{b}(\hat{X})$ be defined by $(L f)(x, y)=f(x)$, for $(x, y) \in \hat{X}$. Clearly, $L$ is an isometry. Let the representation $r$ of $A=C_{b}(X)$ on $\mathcal{H}$ be $r=M L$, that is $(r(f) \xi)(x, y)=f(x) \xi(x, y)$, for $f \in A, \xi \in \mathcal{H}$, and $(x, y) \in \hat{X}$. Since $L$ and $M$ are isometries, so is $r$. If $D: \mathcal{H} \rightarrow \mathcal{H}$ is defined by $(D \xi)(x, y)=\xi(y, x) / d(x, y)$, then $D$ is an unbounded operator on $\mathcal{H}$. 
Furthermore, for $f \in A, \xi \in \mathcal{H}$, and $(x, y) \in \hat{X}$,

$$
\begin{aligned}
([D, r(f)] \xi)(x, y) & =((D r(f)-r(f) D) \xi)(x, y) \\
& =(D(r(f) \xi))(x, y)-(r(f)(D \xi))(x, y) \\
& =\frac{(r(f) \xi)(y, x)}{d(x, y)}-f(x)(D \xi)(x, y) \\
& =\frac{f(y) \xi(y, x)}{d(x, y)}-f(x) \frac{\xi(y, x)}{d(x, y)}=\frac{f(y)-f(x)}{d(x, y)} \xi(y, x) .
\end{aligned}
$$

With $D_{d}$ denoting the de Leeuw derivation $D_{d}: C_{b}(X) \rightarrow C_{b}(\hat{X})$, and $F: \mathcal{H} \rightarrow \mathcal{H}$ the flip operator, $(F \xi)(x, y)=\xi(y, x)$, for $\xi \in \mathcal{H}$ and $(x, y) \in \hat{X}$, we see that $T f=[D, r(f)]=M\left(D_{d}(f)\right) F$, for $f \in A$. Clearly, $\|[D, r(f)]\|=$ $\left\|M\left(D_{d}(f)\right)\right\|=\left\|D_{d}(f)\right\|=\mathrm{p}(f)$, the Lipschitz norm of $f$. Thus, $\{f$ : $[D, r(f)]$ extends to a bounded operator $\}$ is precisely the algebra of Lipschitz functions, and $\overline{\operatorname{Lip}(X, d)}=C_{b u}(X)$, the uniformly continuous functions on $X$. Thus, we have the triple $(A, \mathcal{H}, D)$ as in Connes's definition satisfying $(\mathrm{C} 1)$ and $(\mathrm{C} 2)$. However, (C3) - which is $\left(1+D^{2}\right)^{-1}$ is a compact operator - will not usually be satisfied. Note that there is flexibility in choosing $\mu$ in the above construction. For example, it can be taken to be a discrete measure on $\hat{X}$, so that $L^{2}(\hat{X}, \mu)=l^{2}(\hat{X})$. Or we can take any full non-atomic measure $\nu$ on $X$ (so that $\nu \times \nu(\Delta)=0$ ), and set $\mu=\nu \times \nu$.

\section{Necessary and sufficient conditions for $\mathcal{T}_{d_{T}}=\mathcal{T}$.}

We present first necessary and sufficient conditions for $\mathcal{T}_{d_{T}}=\mathcal{T}$ (Theorem 5.1) which are similar to Theorem 2.2 and which are a generalization of Theorem 4.6. Then, we present other necessary and sufficient conditions for $\mathcal{T}_{d_{T}}=\mathcal{T}$ which are connected to the de Leeuw derivation defined by $d_{T}$ (Theorem 5.3), by using the results of the previous section.

Theorem 5.1. Let $(X, \mathcal{T})$ be a topological space, and let $B$ be a normed space. Let $T$ be a linear operator with $\operatorname{Dom}(T) \subseteq C_{b}(X)$, and Range $(T) \subseteq$ $B$, which satisfies: $(\mathrm{O} 1) \operatorname{Dom}(T)$ separates points of $X$; and $(\mathrm{O} 2) \operatorname{ker} T=$ $\mathbb{C} 1$. Let $d_{T}$ be defined by $d_{T}(x, y)=\sup \{|f(x)-f(y)|: f \in \operatorname{Dom}(T) \&\|T f\|$ $\leq 1\}$ for $x, y \in X$. Let for $z \in X, M(z)=\{f: f(z)=0\}$; let $Q$ denote the quotient operator $Q: \operatorname{Dom}(T) \rightarrow \operatorname{Dom}(T) / \mathbb{C} 1$; and let $\hat{T}$ be the induced one-to-one operator $\operatorname{Dom}(T) / \mathbb{C} 1 \rightarrow B$. The following are equivalent:

(1) $d_{T}$ is a metric on $X$, and $\left(X, d_{T}\right)$ is precompact.

(2) $\overline{\{f \in \operatorname{Dom}(T):\|T f\| \leq 1\} / \mathbb{C} 1}$ is compact. Equivalently, $\hat{T}^{-1}$ is compact.

(3) For any fixed $z \in X$, the closure of $B_{1}=\{f \in \operatorname{Dom}(T): f \in$ $M(z) \&\|T f\| \leq 1\}$ is compact (in the norm topology). Equivalently, $\left(\left.T\right|_{M(z)}\right)^{-1}$ is compact. 
Furthermore, if a sequence $\left(f_{n}\right)_{n=1}^{\infty} \subseteq B_{1}$ converges uniformly to $f$, then $f \in \operatorname{Lip}\left(X, d_{T}\right)$, and $\mathrm{p}(f) \leq 1$.

Proof. Recall that an operator is compact if the image of the unit ball under that operator is precompact.

$(3) \Rightarrow(1)$ Suppose that $B_{1}=\{f \in \operatorname{Dom}(T): f \in M(z) \&\|T f\| \leq 1\}$ is precompact, that is, $\overline{B_{1}}$ is compact. Since it is bounded, by Theorem 2.2 , $d_{T}$ is a bounded metric on $X$. In particular, $d_{T}$ is a metric. Let $\left(x_{n}\right)_{n=1}^{\infty}$ be any sequence in $X$. We will show that it has a Cauchy subsequence, and so $\left(X, d_{T}\right)$ is precompact.

Fix $\varepsilon>0$. Since $\overline{B_{1}}$ is compact, there exist $N$ and $f_{1}, \ldots, f_{N} \in B_{1}$ such that for all $f \in \overline{B_{1}},\left\|f-f_{k}\right\|<\varepsilon / 8$, for some $k \leq N$. For any $x, y \in X$, there is $f \in B_{1}$ such that $d_{T}(x, y)<|f(x)-f(y)|+\varepsilon / 8$. Let $k$ be such that $\left\|f-f_{k}\right\|<\varepsilon / 8$. Then $|f(x)-f(y)| \leq\left|f(x)-f_{k}(x)\right|+\left|f_{k}(x)-f_{k}(y)\right|+$ $\left|f_{k}(y)-f(y)\right|<\left|f_{k}(x)-f_{k}(y)\right|+2 \varepsilon / 8$. What we get is that for all $x, y \in X$ there exists $k \leq N$ such that $d_{T}(x, y)<\left|f_{k}(x)-f_{k}(y)\right|+3 \varepsilon / 8$.

Consider $N$ sequences of complex numbers $\left(f_{k}\left(x_{n}\right)\right)_{n=1}^{\infty}, k=1, \ldots, N$. They are bounded, since each $f_{k}$ is a bounded function. So there is a subsequence $\left(x_{n_{i}}\right)_{i=1}^{\infty}$ such that all the subsequences $\left(f_{k}\left(x_{n_{i}}\right)\right)_{i=1}^{\infty}$ are convergent, say to $z_{k} \in \mathbb{C}$. In particular, for any $\varepsilon>0$ there exists $J$ such that $j \geq J$ implies $\left|f_{k}\left(x_{n_{j}}\right)-z_{k}\right|<\varepsilon / 8$, for all $k$, and so $i, j \geq J$ implies $\left|f_{k}\left(x_{n_{i}}\right)-f_{k}\left(x_{n_{j}}\right)\right|<2 \varepsilon / 8$ for all $k$. With the previous observation, this means that $i, j \geq J$ implies $d_{T}\left(x_{n_{i}}, x_{n_{j}}\right)<\left|f_{k}\left(x_{n_{i}}\right)-f_{k}\left(x_{n_{j}}\right)\right|+3 \varepsilon / 8$ $<2 \varepsilon / 8+3 \varepsilon / 8<\varepsilon$.

We can construct the desired $d_{T}$-Cauchy subsequence $\left(y_{k}\right)_{k=1}^{\infty}$ of $\left(x_{n}\right)_{n=1}^{\infty}$ recursively in the following way. Let $\varepsilon_{n}=1 / 2^{n}, n \geq 1$. First, we construct subsequences $\left(v_{k l}\right)_{l=1}^{\infty}$ of $\left(x_{n}\right)_{n=1}^{\infty}$, for $k \geq 1$, with the property that for $i, j>$ $k, d_{T}\left(v_{k i}, v_{k j}\right)<\varepsilon_{k}$. Let $v_{11}=x_{1}$. Let $\left(u_{s}\right)_{s=1}^{\infty}$ be a subsequence of $\left(x_{n}\right)_{n=1}^{\infty}$ constructed in the previous paragraph, for $\varepsilon=\varepsilon_{1}$, and with $J$ such that $i, j \geq J$ implies $d_{T}\left(u_{s_{i}}, u_{s_{j}}\right)<\varepsilon_{1}$ as above. Let $v_{1 l}=v_{J+l}$ for $l>1$, so that for $i, j>k, d_{T}\left(v_{1 i}, v_{1 j}\right)<\varepsilon_{1}$. suppose we have constructed $\left(v_{k l}\right)_{l=1}^{\infty}$ for $k=$ $1, \ldots, K-1$. Let $v_{K l}=v_{(K-1) l}$ for $l=1, \ldots, K$. Let $\left(u_{s}\right)_{s=1}^{\infty}$ be a subsequence of $\left(v_{(K-1) l}\right)_{l=1}^{\infty}$, and $J$ be such that $i, j \geq J$ implies $d_{T}\left(u_{s_{i}}, u_{s_{j}}\right)<\varepsilon_{K}$. Let $v_{K l}=u_{J+l-K}$ for $l>K$, so that $i, j>K$ implies $d_{T}\left(v_{K i}, v_{K j}\right)<\varepsilon_{K}$. This produces the desired subsequences $\left(v_{k l}\right)_{l=1}^{\infty}, k \geq 1$. Clearly, $\left(y_{k}\right)_{k=1}^{\infty}$ defined by $y_{k}=v_{k k}$ satisfies that $i, j>k$ implies $d_{T}\left(y_{i}, y_{j}\right)<\varepsilon_{k}$.

We conclude that $\left(X, d_{T}\right)$ is precompact.

$(1) \Rightarrow(3)$ Let $\left(X^{\prime}, d_{T}\right)$ be the completion of $\left(X, d_{T}\right)$, which is compact. Since any function $f \in B_{1}$ is uniformly continuous on $X$, it extends uniquely in a norm preserving way to a continuous function on $X^{\prime}$. Let $B_{1}^{\prime}=\left\{f^{\prime}\right.$ : $\left.f \in B_{1}\right\}$. Since $f^{\prime} \in B_{1}^{\prime}$ implies $\left|f^{\prime}(x)-f^{\prime}(y)\right| \leq d_{T}(x, y)$ for all $x, y \in X^{\prime}$, $B_{1}^{\prime}$ is an equicontinuous family of functions. Furthermore, for any $x \in X^{\prime}$, and $f^{\prime} \in B_{1}^{\prime}$, we have that $\left|f^{\prime}(x)\right| \leq d_{T}(z, x) \leq d_{X}$, the diameter of $X$, so it 
is bounded. By Arzela - Ascoli theorem, any sequence in $B_{1}^{\prime}$, and hence in $B_{1}$, has a subsequence which converges uniformly to a continuous function on $X^{\prime}$, so to a bounded continuous function on $X$. We conclude that $\overline{B_{1}}$ is compact.

$(2) \Leftrightarrow(3)$ We showed in the proof of Theorem 2.2 that $\left.Q\right|_{M(z)}$ is oneto-one onto bicontinuous, with $\left\|\left.Q\right|_{M(z)}\right\| \leq 1$ and $\left\|\left(\left.Q\right|_{M(z)}\right)^{-1}\right\| \leq 2$. The equivalence of (2) and (3) follows directly.

We show now that if $\left(f_{n}\right)_{n=1}^{\infty} \subseteq B_{1}$ converges uniformly to $f$, then $f \in$ $\operatorname{Lip}\left(X, d_{T}\right)$, and $\mathrm{p}(f) \leq 1$. Fix any $x, y \in X$, let $\varepsilon>0$, and let $N$ be such that $n>N$ implies $\left\|f-f_{n}\right\|<\varepsilon / 2$. Then $|f(x)-f(y)| \leq\left|f(x)-f_{n}(x)\right|+$ $\left|f_{n}(x)-f_{n}(y)\right|+\left|f_{n}(y)-f(y)\right|<\left|f_{n}(x)-f_{n}(y)\right|+\varepsilon \leq d_{T}(x, y)+\varepsilon$, and we conclude that $|f(x)-f(y)| \leq d_{T}(x, y)$, as desired. In particular, it shows that $B_{1 D_{d_{T}}}=\left\{f \in \operatorname{Lip}\left(X, d_{T}\right): \mathrm{p}(f) \leq 1\right\}$ is compact if $\left(X, d_{T}\right)$ is precompact, which is a variation of the conclusions of Theorem 4.6.

Corollary 5.2. Let $(X, \mathcal{T})$ be a compact Hausdorff space, and let $B$ be a normed space. Let $T$ be a linear operator with $\operatorname{Dom}(T) \subseteq C(X)$, and Range $(T) \subseteq B$, which satisfies: (O1) $\operatorname{Dom}(T)$ separates points of $X$; and (O2) $\operatorname{ker} T=\mathbb{C} 1$. Let $d_{T}$ be defined by $d_{T}(x, y)=\sup \{|f(x)-f(y)|: f \in$ $\operatorname{Dom}(T) \&\|T f\| \leq 1\}$ for $x, y \in X$. The following are equivalent:

(1) $d_{T}$ is a metric on $X$, and $\mathcal{T}_{d_{T}}=\mathcal{T}$.

(2) $\overline{\{f \in \operatorname{Dom}(T):\|T f\| \leq 1\} / \mathbb{C} 1}$ is compact. Equivalently, $\hat{T}^{-1}$ is compact.

(3) For any fixed $z \in X$, the closure of $B_{1}=\{f \in \operatorname{Dom}(T): f \in$ $M(z) \&\|T f\| \leq 1\}$ is compact (in the norm topology). Equivalently, $\left(\left.T\right|_{M(z)}\right)^{-1}$ is compact.

Proof. (2) $\Leftrightarrow(3)$ is the same as in the theorem. For $(1) \Rightarrow(2)$ or (3), if $\mathcal{T}_{d_{T}}=\mathcal{T}$, then $\left(X, d_{T}\right)$ is compact, and (2) and (3) follow from the theorem. Conversely, if (2) and (3) hold, then by the theorem, $\left(X, d_{T}\right)$ is precompact. But, by Proposition $3.1,\left(X, d_{T}\right)$ is always complete, so in fact $\left(X, d_{T}\right)$ is compact, and again by the same proposition, $\mathcal{T}_{d_{T}}=\mathcal{T}$.

We now present necessary and sufficient conditions for $\mathcal{T}_{d_{T}}=\mathcal{T}$ which follow immediately from the results about de Leeuw derivations.

Theorem 5.3. Let $(X, \mathcal{T})$ be a compact Hausdorff space, and let $B$ be a normed space. Let $T$ be a linear operator with $\operatorname{Dom}(T) \subseteq C(X)$, $\operatorname{ker} T=\mathbb{C} 1$, and Range $(T) \subseteq B$, which defines the metric $d_{T}$ on the set $X$.

(a) If $\mathcal{T}_{d_{T}}=\mathcal{T}$ then $T$ is unbounded on every infinite dimensional subspace of its domain.

(b) If $D$ is the de Leeuw derivation associated to $T$, i.e. $d$ is defined by $d_{T}$, and if $D$ is unbounded on every infinite dimensional subspace of its domain, then $\mathcal{T}_{d_{T}}=\mathcal{T}$. 
Proof. (a) Suppose that $\mathcal{T}_{d_{T}}=\mathcal{T}$. By Proposition 4.5, for all $f \in \operatorname{Dom}(T)$, $\|T f\| \geq\|\hat{D} f\|$. By Theorem 4.7, $\hat{D}$, and hence $T$, is unbounded on every infinite dimensional subspace of its domain.

(b) By Proposition $4.3, d_{D}=d_{T}$. By Theorem $4.7,\left(X, d_{D}\right)$ is precompact, and hence so is $\left(X, d_{T}\right)$. But, by Proposition $3.1,\left(X, d_{T}\right)$ is complete, so in fact compact. Since by Proposition $3.1, \mathcal{T} \subseteq \mathcal{T}_{d_{T}}$, and both are compact Hausdorff, we conclude that $\mathcal{T}_{d_{T}}=\mathcal{T}$.

Clearly, the above theorem could be also stated as: $\mathcal{T}_{d_{T}}=\mathcal{T}$ if and only if the de Leeuw derivation associated to $T$ is unbounded on every infinite dimensional subspace of its domain.

Next, we show that the condition:

(O4): $T$ is unbounded on every infinite dimensional subspace of its domain,

is only necessary, but not sufficient for $\mathcal{T}_{d_{T}}=\mathcal{T}$. For the examples which show that, we need some results about Banach spaces $c_{0}$ and $l_{1}$, which we quote from [7].

Theorem 5.4. ([7], Theorem I.2.5.) Let $X$ be either $c_{0}$ or $l_{p}, 1 \leq p<\infty$. Then every infinite dimensional subspace $Y$ of $X$ contains a subspace $Z$ which is isomorphic to $X$ and complemented in $X$ (and, therefore, also in $Y)$.

Theorem 5.5. ([7], Theorem I.2.7.) Assume that $1 \leq p<r<\infty$. Then every bounded linear operator from $l_{r}$ to $l_{p}$ is compact. The same is true for linear operators from $c_{0}$ to $l_{p}$. Consequently, no space of the class $l_{p}$, $1 \leq p<\infty$, and $c_{0}$ is isomorphic to a subspace of another member of this class.

We write down an easy consequence of these results which will be used.

Proposition 5.6. Let $1 \leq p<\infty$. There is no infinite dimensional subspace $V$ of $l_{p}$ such that $\exists M>0$ satisfying $\|f\|_{p} /\|f\|_{\infty} \leq M \forall f \in V$.

Proof. Suppose that the claim is not true. Then there exists an infinite dimensional subspace $V$ of $l_{p}$ satisfying the above condition. By Theorem I.2.5 of [7] (quoted above), there exists an infinite dimensional subspace $W$ of $V$ which is isomorphic to $l_{p}$ and complemented in $l_{p}$ (and so also in $V$ ). Let $T: l_{p} \rightarrow W$ be the isomorphism map. Let $S=i T: l_{p} \rightarrow c_{0}$, where $i$ is the inclusion map $i: W \rightarrow c_{0}, i(f)=f$. Clearly, $S$ is one-to-one, and $\|S\| \leq\|T\|\|i\|=\|T\|$. Let $Z=\operatorname{Range}(S)$. Then $S^{-1}: Z \rightarrow l_{p}$ and $S^{-1}=T^{-1} i^{-1}$, and so $\left\|S^{-1}\right\| \leq\left\|T^{-1}\right\| M$. So $S$ is an isomorphism of $l_{p}$ with a subspace of $c_{0}$. By Theorem I.2.7 of [7] (quoted above), that is not possible, so we get the desired contradiction. 
Example 5.7. This is an example of a closed operator $T$ which is unbounded on every infinite dimensional subspace of its domain, yet $\mathcal{T}_{d_{T}} \neq \mathcal{T}$. Let $X=\left\{x_{n}: n \in \mathbb{N}\right\} \cup\left\{x_{0}\right\}$, with $x_{0}$ the only accumulation point, and $x_{n} \rightarrow x_{0}$ as $n \rightarrow \infty$. For $n \geq 1$, let $e_{n} \in C(X)$ be defined by $e_{n}\left(x_{k}\right)=1$ if $k=n$, and $e_{n}\left(x_{k}\right)=0$ if $k \neq n$. Clearly $\overline{\operatorname{span}\left(e_{n}\right)}=M\left(x_{0}\right)=\{f \in C(X)$ : $\left.f\left(x_{n}\right) \rightarrow 0\right\}=c_{0}$. Let $1 \leq p<\infty$, and let $\operatorname{Dom}(T)=l_{p}\left(\left\{x_{k}: k \geq 1\right\}\right)$. Let $T: \operatorname{Dom}(T) \rightarrow l_{p}(\mathbb{N})$ be defined by $T f=f$. So $\left\|T e_{n}\right\|=1$. It is easily checked that $d_{T}\left(x_{n}, x_{k}\right)=1$ for all different $k, n \geq 0$, by taking first functions in the $\operatorname{span}\left(e_{n}\right)$, and then concluding by Remark 2.3, that the metric stays the same when we include all the functions in the domain of the closure of the operator (which is here equal to $\operatorname{Dom}(T)$ ).

Let $V$ be any infinite dimensional subspace of $\operatorname{Dom}(T)$. By Proposition 5.6 , there is no such constant $M$ for which $\|T f\| /\|f\|=\|f\|_{p} /\|f\|_{\infty} \leq M$. Thus $T$ is unbounded on $V$.

We also show that the ratio $\|T f\| / \mathrm{p}(f)$ is unbounded on every infinite dimensional subspace of $\operatorname{Dom}(T)$. Note that since $d(x, y)=1$ for all $x, y \in X$, $\mathrm{p}(f)=\sup \{|f(x)-f(y)| / d(x, y): x, y \in X\}=\sup \{|f(x)-f(y)|\} \leq$ $2\|f\|_{\infty}$. This in particular shows that $\operatorname{Lip}(X, d)=C(X)$. Also, $\|T f\| / \mathrm{p}(f)=$ $(\|T f\| /\|f\|)(\|f\| / \mathrm{p}(f)) \geq(1 / 2)(\|T f\| /\|f\|)$, and so $\|T f\| / \mathrm{p}(f)$ is unbounded on every infinite dimensional subspace of $\operatorname{Dom}(T)$.

Example 5.8. This is an example of an operator $T$ which is unbounded on every infinite dimensional subspace of its domain, with $\mathcal{T}_{d_{T}}=\mathcal{T}$, and $\|T f\| / \mathrm{p}(f)$ also unbounded on every infinite dimensional subspace of $\operatorname{Dom}(T)$. Let $X=\left\{x_{n}: n \in \mathbb{N}\right\} \cup\left\{x_{0}\right\}$, with $x_{0}$ the only accumulation point, and $x_{n} \rightarrow x_{0}$ as $n \rightarrow \infty$. For $n \geq 1$, let $e_{n} \in C(X)$ be defined by $e_{n}\left(x_{k}\right)=1$ if $k=n$, and $e_{n}\left(x_{k}\right)=0$ if $k \neq n$. Let $f_{n}=e_{n} / n^{2}$. Clearly $\operatorname{span}\left(f_{n}\right)=\operatorname{span}\left(e_{n}\right)$, and so $\overline{\operatorname{span}\left(f_{n}\right)}=M\left(x_{0}\right)=\left\{f \in C(X): f\left(x_{n}\right) \rightarrow 0\right\}$. Let $\operatorname{Dom}(T)=\operatorname{span}\left(f_{n}\right)$. Let $T: \operatorname{Dom}(T) \rightarrow l_{1}(\mathbb{N})$ be defined by $T f_{n}=e_{n}$. It is easily checked that $d_{T}\left(x_{n}, x_{k}\right)=1 / n^{2}$ for all $k>n \geq 1$ and that $d_{T}\left(x_{n}, x_{0}\right)=1 / n^{2}$ for all $n \geq 1$.

If $f \in \operatorname{Dom}(T)$ then $f=\sum_{j=1}^{m} a_{j} f_{j}=\sum_{j=1}^{m} a_{j}\left(e_{j} / n^{2}\right)$. Thus $T f=$ $\sum_{j=1}^{m} a_{j} T f_{j}=\sum_{j=1}^{m} a_{j} e_{j}$, and so $\|T f\|=\sum_{j=1}^{m}\left|a_{j}\right|$. On the other hand, $\|f\|_{\infty}=\sup \left\{\left|\sum_{j=1}^{m} a_{j} f_{j}\left(x_{n}\right)\right|: n \geq 1\right\}=\sup \left\{\left|a_{n} / n^{2}\right|: 1 \leq n \leq m\right\} \leq$ $\sup \left\{\left|a_{n}\right|: 1 \leq n \leq m\right\}$. We have

$$
\frac{\|T f\|}{\|f\|_{\infty}}=\frac{\sum_{j=1}^{m}\left|a_{j}\right|}{\sup \left\{\left|a_{n} / n^{2}\right|: 1 \leq n \leq m\right\}} \geq \frac{\sum_{j=1}^{m}\left|a_{j}\right|}{\sup \left\{\left|a_{n}\right|: 1 \leq n \leq m\right\}} .
$$

Let $V$ be any infinite dimensional subspace of $\operatorname{Dom}(T)$. By Proposition 5.6 , there is no constant $M$ such that $\left(\sum_{j=1}^{m}\left|a_{j}\right| / \sup \left\{\left|a_{n}\right|: 1 \leq n \leq m\right\}\right) \leq$ $M$. Thus $T$ is unbounded on $V$. 
We show that the ratio $\|T f\| / \mathrm{p}(f)$ is unbounded on every infinite dimensional subspace of $\operatorname{Dom}(T)$. Note that

$$
\begin{aligned}
\mathrm{p}(f) & =\sup \left\{\frac{\left|f\left(x_{l}\right)-f\left(x_{k}\right)\right|}{d\left(x_{l}, x_{k}\right)}: l>k\right\} \\
& =\sup \left\{k^{2}\left|f\left(x_{l}\right)-f\left(x_{k}\right)\right|: l>k\right\}=\sup \left\{k^{2}\left|\frac{1}{l^{2}} a_{l}-\frac{1}{k^{2}} a_{k}\right|: l>k\right\} \\
& =\sup \left\{\left|\frac{k^{2}}{l^{2}} a_{l}-a_{k}\right|: l>k\right\} \leq 2 \sup \left\{\left|a_{j}\right|: 1 \leq j \leq m\right\} .
\end{aligned}
$$

Thus

$$
\frac{\|T f\|}{\mathrm{p}(f)} \geq \frac{1}{2} \frac{\sum_{j=a}^{m}\left|a_{j}\right|}{\sup \left\{\left|a_{j}\right|: 1 \leq j \leq m\right\}} .
$$

So $\|T f\| / \mathrm{p}(f)$ is unbounded on every infinite dimensional subspace of $\operatorname{Dom}(T)$.

\section{Defining a metric on the state space of a $C^{*}$-algebra.}

We turn our attention to noncommutative $C^{*}$-algebras. In fact, we generalize our results in two directions: (1) from $C(X)$ to any unital $C^{*}$-algebra - that is, from commutative to the noncommutative case; $(2)$ from a metric on a compact Hausdorff space $X$ to an extension of that metric to the space of the probability measures on $X$, which in the noncommutative case means the extension of the metric from the pure state space to the state space.

The discussion and notation follows that of [6], Section 4.3. Let $A$ be any unital $C^{*}$-algebra. We use the following notation: $\mathcal{S}(A)$ for the state space of $A$, the set of all positive linear functionals $\rho$ on $A$ such that $\rho(\mathrm{I})=1$; $\mathcal{P}(A)$ for the set of all pure states of $A$, which is the set of extreme points of $\mathcal{S}(A) ; \overline{\mathcal{P}(A)}=\overline{\mathcal{P}(A)}{ }^{w^{*}}$ for the pure state space of $A$, the weak-* closure of $\mathcal{P}(A)$. Since $\mathcal{S}(A)$ is compact for the weak-* topology, by the Krein-Milman theorem it is the closed convex hull of its extreme points, which means that $\mathcal{S}(A)=\overline{\mathrm{co}}(\mathcal{P}(A))$. In the case when $A=C(X)$ for $X$ compact Hausdorff, we have $X$ "=" $\mathcal{P}(A)=\overline{\mathcal{P}(A)}$, and the weak-* topology on $X$ coincides with the original one. In this case $\mathcal{S}(A)=\mathcal{P}(X)$, the set of probability measures on $X$. However, in general, $\mathcal{P}(A)$ is not closed.

Recall that a function representation of a unital $C^{*}$-algebra $A$ on a compact Hausdorff space $X$ is a linear map $\Phi: A \rightarrow C(X)$, such that $\Phi \mathrm{I}=1$ (i.e. $\Phi \mathrm{I}(x)=1 \forall x \in X)$, and $\Phi a \in C(X)^{+}$if and only if $a \in A^{+}$. If for all $x, y \in X$ there exists $a \in A$ such that $\Phi(x) \neq \Phi(y)$, then $\Phi$ is a separating function representation. Furthermore, $\Phi$ is an isometry on $A_{s a}$, the selfadjoint elements of $A$, and for any $a \in A,\|a\| / 2 \leq\|\Phi a\|_{\infty} \leq\|a\|$. So $\Phi$ is a one-to-one bicontinuous operator, which means that it is a Banach space isomorphism of $A$ with $\Phi(A)$.

Let $X$ be a closed subset of $\mathcal{S}(A)$ containing all the pure states, $\mathcal{P}(A) \subseteq$ $X \subseteq \mathcal{S}(A)$. Consider the canonical function representation $\Phi$ of $A$ on 
$X$, a linear map $\Phi: A \rightarrow C(X)$ defined by $(\Phi a)(\rho)=\rho(a)$ for $\rho \in X$. Clearly, we have: (1) $\Phi \mathrm{I}=1$; (2) $\Phi a \in C(X)^{+}$if and only if $a \in A^{+}$; and (3) $\Phi$ is separating, since for any two distinct elements $\rho, \sigma \in X$ there exists an element $a \in A$ such that $\rho(a) \neq \sigma(a)$. This special function representation is the one we are going to use, and it is called the canonical, or Kadison's function representation. In general $\Phi$ is not multiplicative, so it is not an algebra homomorphism. However, as it is well known, if $A$ is commutative and $X=\mathcal{P}(A)=\overline{\mathcal{P}(A)}$, then $\Phi$ is the Gelfand transform, which is multiplicative, and in fact an isometric isomorphism of two algebras, $A$ and $C(\mathcal{P}(A))$.

Using the canonical function representation we can obtain noncommutative versions of Theorem 2.2, Corollary 5.2, and Theorem 5.3. The following simple observation is crucial for passing from commutative to the noncommutative case.

Proposition 6.1. Let $A$ be a unital $C^{*}$-algebra, $X$ a closed subset of $\mathcal{S}(A)$ containing $\mathcal{P}(A)$, and $\mathcal{T}_{w^{*}}$ the weak-* topology on $X$. Let $\Phi$ be the canonical function representation of $A$ on $X$. Let $T$ be an operator with $\operatorname{Dom}(T) \subseteq A$, and Range $(T) \subseteq B$, where $B$ is a normed space. Let $d_{T}$ be defined by the formula

$$
d_{T}(\phi, \psi)=\sup \{|\phi(a)-\psi(a)|: a \in \operatorname{Dom}(T) \&\|T a\| \leq 1\},
$$

for $\phi, \psi \in X$. Let $S: C(X) \rightarrow B$ be the operator with $\operatorname{Dom}(S)=\Phi(\operatorname{Dom}(T))$ and Range $(S)=$ Range $(T)$, defined by $S=T \Phi^{-1}$. With $d_{S}$ defined as usual in the commutative case, we have that $d_{T}=d_{S}$, and so:

(a) $\operatorname{Dom}(T)$ separates points of $X$ if and only if $\operatorname{Dom}(S)$ does, and $\operatorname{ker} T=$ $\mathbb{C} 1$ if and only if $\operatorname{ker} S=\mathbb{C} 1 .\{a \in \operatorname{Dom}(T):\|T a\| \leq 1\} / \mathbb{C} 1$ is bounded if and only if $\{f \in \operatorname{Dom}(S):\|T f\| \leq 1\} / \mathbb{C} 1$ is bounded. $\{a \in \operatorname{Dom}(T)$ : $\|T a\| \leq 1\} / \mathbb{C} 1$ is precompact if and only if $\{f \in \operatorname{Dom}(S):\|T f\| \leq 1\} / \mathbb{C} 1$ is precompact.

(b) (1) $d_{T}$ is a metric on $X$ if and only if $d_{S}$ is; (2) $d_{T}$ is a bounded metric on $X$ if and only if $d_{S}$ is; (3) $\mathcal{T}_{d_{T}}=\mathcal{T}_{w^{*}}$ if and only if $\mathcal{T}_{d_{S}}=\mathcal{T}_{w^{*}}$.

Proof. (a) is the consequence of the fact that $\Phi$ is one-to-one bicontinuous.

(b) We have for $\phi, \psi \in X$

$$
\begin{aligned}
d_{S}(\phi, \psi) & =\sup \{|f(\phi)-f(\psi)|: f \in \operatorname{Dom}(S) \&\|S f\| \leq 1\} \\
& =\sup \left\{|\Phi a(\phi)-\Phi a(\psi)|: a \in \operatorname{Dom}(T) \&\left\|T \Phi^{-1} \Phi a\right\|=\|T a\| \leq 1\right\} \\
& =\sup \{|\phi(a)-\psi(a)|: a \in \operatorname{Dom}(T) \&\|T a\| \leq 1\}=d_{T}(\phi, \psi) .
\end{aligned}
$$

So $d_{S}=d_{T}$, and (b) follows.

The next theorem is the noncommutative version of Theorem 2.2.

Theorem 6.2. Let $A$ be a unital $C^{*}$-algebra, and let $X$ be a closed subset of $\mathcal{S}(A)$ containing $\mathcal{P}(A)$. Let $T$ be an operator with $\operatorname{Dom}(T) \subseteq A$, Range $(T) \subseteq$ 
$B$, where $B$ is a normed space, which satisfies: (O1) Dom $(T)$ separates points of $X$; and $(\mathrm{O} 2) \operatorname{ker} T=\mathbb{C} 1$. Let $d_{T}$ be defined by the formula (5). Let $Q$ denote the quotient operator $Q: \operatorname{Dom}(T) \rightarrow \operatorname{Dom}(T) / \mathbb{C} 1$, and let $\hat{T}$ be the induced one-to-one operator $\operatorname{Dom}(T) / \mathbb{C} 1 \rightarrow B$. The following are equivalent:

(1) $d_{T}$ is a bounded metric on $X$.

(2) $\{a \in \operatorname{Dom}(T):\|T a\| \leq 1\} / \mathbb{C} 1$ is bounded. Equivalently, $\hat{T}^{-1}$ is bounded.

Proof. The claim is immediate from Theorem 2.2 and Proposition 6.1.

The following is the noncommutative version of Corollary 5.2.

Theorem 6.3. Let $A$ be a unital $C^{*}$-algebra, $X$ a closed subset of $\mathcal{S}(A)$ containing $\mathcal{P}(A)$, and $\mathcal{T}_{w^{*}}$ the weak- ${ }^{*}$ topology on $X$. Let $T$ be an operator with $\operatorname{Dom}(T) \subseteq A$, Range $(T) \subseteq B$, where $B$ is a normed space, which satisfies: (O1) $\operatorname{Dom}(T)$ separates points of $X$; and $(\mathrm{O} 2) \operatorname{ker} T=\mathbb{C} 1$. Let $d_{T}$ be defined by the formula (5). Let $Q$ denote the quotient operator $Q: \operatorname{Dom}(T) \rightarrow \operatorname{Dom}(T) / \mathbb{C} 1$, and let $\hat{T}$ be the induced one-to-one operator $\operatorname{Dom}(T) / \mathbb{C} 1 \rightarrow B$. The following are equivalent:

(1) $d_{T}$ is a metric on $X$, and $\mathcal{T}_{d_{T}}=\mathcal{T}_{w^{*}}$.

(2) $\overline{\{a \in \operatorname{Dom}(T):\|T a\| \leq 1\} / \mathbb{C} 1}$ is compact. Equivalently, $\hat{T}^{-1}$ is compact.

Proof. The equivalence of (1) and(2) is immediate from Proposition 6.1 and Corollary 5.2.

Theorem 5.3 has also its noncommutative analog.

Theorem 6.4. Let $A$ be a unital $C^{*}$-algebra, $X$ a closed subset of $\mathcal{S}(A)$ containing $\mathcal{P}(A)$, and $\mathcal{T}_{w^{*}}$ the weak-* topology on $X$. Let $T$ be an operator with $\operatorname{Dom}(T) \subseteq A$, Range $(T) \subseteq B, B$ a normed space, and suppose that $T$ defines a metric $d_{T}$ on $X$ by the formula (5).

(a) If $\mathcal{T}_{d_{T}}=\mathcal{T}_{w^{*}}$ then $T$ is unbounded on every infinite dimensional subspace of its domain.

(b) If $D$ is the de Leeuw derivation defined by $d_{T}$, and if $D$ is unbounded on every infinite dimensional subspace of its domain, then $\mathcal{T}_{d_{T}}=\mathcal{T}_{w^{*}}$.

Proof. (a) This follows from Proposition 6.1 and Theorem 5.3 (a).

(b) This is exactly the same as in Theorem 5.3 (b).

As in the commutative case, the above theorem could be stated as: $\mathcal{T}_{d_{T}}=$ $\mathcal{T}_{w^{*}}$ if and only if the de Leeuw derivation associated to $T$ is unbounded on every infinite dimensional subspace of its domain.

In most examples $\operatorname{Dom}(T)$ separates points of $\mathcal{S}(A)$ (however, it is not true that if $\operatorname{Dom}(T)$ separates points of $\overline{\mathcal{P}(A)}$, it does so on all of $\mathcal{S}(A)$ ). Usually, $\overline{\operatorname{Dom}(T)}=A$, as is the case when $T$ is defined from an unbounded 
Fredholm module (see the introduction), and in such a case it is easy to see that $\operatorname{Dom}(T)$ separates points of $\mathcal{S}(A)$. In cases like that it is natural to ask whether, if $T$ defines an appropriate metric on $\overline{\mathcal{P}(A)}$, whether it does so on all of $\mathcal{S}(A)$.

Theorem 6.5. Let $A$ be a unital $C^{*}$-algebra, and suppose that $T$ is an operator with $\operatorname{Dom}(T) \subseteq A$ which separates points of $\mathcal{S}(A)$, and Range $(T) \subseteq B$, $B$ a normed space, such that $T$ defines metric $d_{T}$ on $X=\overline{\mathcal{P}(A)}$.

(a) If $d_{T}$ is bounded on $X$, then $T$ defines a bounded metric $d_{T}$ on $\mathcal{S}(A)$ by formula (5).

(b) If $\mathcal{T}_{d_{T}}=\mathcal{T}_{w^{*}}$ on $\overline{\mathcal{P}(A)}$, then $\mathcal{T}_{d_{T}}=\mathcal{T}_{w^{*}}$ on $\mathcal{S}(A)$.

Proof. (a) Since $d_{T}$ is bounded on $\overline{\mathcal{P}(A)}$, by Theorem 2.2, $\{a \in \operatorname{Dom}(T)$ : $\|T a\| \leq 1\} / \mathbb{C} 1$ is bounded, and since $\operatorname{Dom}(T)$ separates points of $\mathcal{S}(A)$, by Theorem $6.3, d_{T}$ is a bounded metric on $\mathcal{S}(A)$.

(b) Since $\mathcal{T}_{d_{T}}=\mathcal{T}_{w^{*}}$ on $\overline{\mathcal{P}(A)},\left(\overline{\mathcal{P}(A)}, d_{T}\right)$ is compact, and so by Theorem 6.3, $\{a \in \operatorname{Dom}(T):\|T a\| \leq 1\} / \mathbb{C} 1$ is precompact. Since $\operatorname{Dom}(T)$ separates points of $\mathcal{S}(A)$, by Theorem 6.3 again, $\mathcal{T}_{d_{T}}=\mathcal{T}_{w^{*}}$ on $\mathcal{S}(A)$.

We give a commutative version of the above theorem.

Corollary 6.6. Let $(X, \mathcal{T})$ be a compact Hausdorff space, and suppose that $T$ is an operator with $\operatorname{Dom}(T) \subseteq C(X)$ which separates points of $\mathcal{P}(X)$, and Range $(T) \subseteq B$, where $B$ is a normed space, such that $T$ defines a metric $d_{T}$ on $X$.

(a) If $d_{T}$ is bounded on $X$, then $T$ defines a bounded metric $d_{T}$ on $\mathcal{P}(X)$, the space of probability measures on $X$ by

$$
d_{T}(\phi, \psi)=\sup \{|\phi(f)-\psi(f)|: f \in \operatorname{Dom}(T) \&\|T f\| \leq 1\},
$$

for $\phi, \psi \in \mathcal{P}(X)$.

(b) If $\mathcal{T}_{d_{T}}=\mathcal{T}$ on $X$, then $\mathcal{T}_{d_{T}}=\mathcal{T}_{w^{*}}$ on $\mathcal{P}(X)$.

Here is the same result phrased in terms of Lipschitz algebras and compact metric spaces.

Corollary 6.7. Let $(X, d)$ be a compact metric space. If the extension of the metric $d$ to $\mathcal{P}(X)$, the space of probability measures on $X$, is given by

$$
d(\phi, \psi)=\sup \{|\phi(f)-\psi(f)|: f \in \operatorname{Lip}(X, d), \mathrm{p}(f) \leq 1\},
$$

for $\phi, \psi \in \mathcal{P}(X)$, then $d$ is a metric on $\mathcal{P}(X)$, and $\mathcal{T}_{d}=\mathcal{T}_{w^{*}}$ on $\mathcal{P}(X)$.

We finally come to our original goal, which is to characterize those unbounded Fredholm modules over a unital $C^{*}$-algebra $A$ whose metric topology coincides with the weak-* topology on $\mathcal{S}(A)$. 
Theorem 6.8. Let $(\mathcal{H}, D)$ be an unbounded Fredholm module over a unital $C^{*}$-algebra $A$, and let the metric d on $\mathcal{S}(A)$ be defined by formula (1), that is

$$
d(\phi, \psi)=\sup \{|\phi(a)-\psi(a)|: a \in A,\|[D, a]\| \leq 1\},
$$

for $\phi, \psi \in \mathcal{S}(A)$.

(1) $d$ is a bounded metric on $\mathcal{S}(A)$ if and only if $\{a \in A:\|[D, a]\| \leq 1\} / \mathbb{C} 1$ is bounded.

(2) $\mathcal{T}_{d}=\mathcal{T}_{w^{*}}$ on $\mathcal{S}(A)$ if and only if $\overline{\{a \in A:\|[D, a]\| \leq 1\} / \mathbb{C} 1}$ is compact.

(3) $\mathcal{T}_{d}=\mathcal{T}_{w^{*}}$ on $\mathcal{S}(A)$ if and only if the de Leeuw derivation $D_{d}$ is unbounded on every infinite dimensional subspace of its domain.

Note that (1) is a slight strengthening of [2], Proposition 4 of Connes.

Proof. Let $T: A \rightarrow \mathcal{B}(\mathcal{H})$ be defined by $T a=[D, a]$ for $a \in A$. By condition (C2) in the Definition 1.1 of an unbounded Fredholm module, $\operatorname{Dom}(T)$ is dense in $A$, hence separates points of $\mathcal{S}(A)$. Also note that the conditions in (1), (2), and (3) imply that $\operatorname{ker} T=\mathbb{C} 1$. So we have that (1) follows from Theorem 6.2, (2) follows from Theorem 6.3, and (3) follows from Theorem 6.4 .

\section{References}

[1] W.G. Bade, P.C. Curtis, Jr. and H.G. Dales, Amenability and weak amenability for Beurling and Lipschitz algebras, Proc. London Math. Soc., 55(3) (1987), 359-377.

[2] A. Connes, Compact metric spaces, Fredholm modules, and hyperfinitness, Ergodic Theory and Dynamical Systems, 9 (1989), 207-220.

[3] _ Noncommutative geometry, Academic Press, San Diego, 1994.

[4] K. de Leeuw, Banach spaces of Lipschitz functions, Studia Mathematica, 21 (1961) 55-66.

[5] J.A. Johnson, Banach spaces of Lipschitz functions and vector-valued Lipschitz functions, Transactions of the AMS, 148 (March 1970), 147-169.

[6] R.V. Kadison and J.R. Ringrose, Fundamentals of the theory of operator algebras, Vol. I, Academic Press, New York, 1983.

[7] J. Lindenstrauss and L. Tzafriri, Classical Banach spaces, Springer-Verlag, Lecture Notes in Mathematics, Vol. 338, Berlin, 1973.

[8] B. Pavlović, Automatic continuity of Lipschitz algebras, Journal of Functional Analysis, 131(1) (1995), 115-144.

[9] _ The category of compact metric spaces and its equivalents, preprint.

[10] , The topology of unbounded Fredholm modules over unital $C^{*}$-algebras, in preparation.

[11] M.A. Rieffel, Comments concerning non-commutative metrics, Talk given at October 1993 AMS meeting at Texas A\&M. 
[12] D.R. Sherbert, Banach algebras of Lipschitz functions, Pacific J. of Math., 13 (1963), 1387-1399.

Received February 5, 1997 and revised January 30, 1998

University of Dublin, Trinity College

Dublin 2, IRELAND

E-mail address: pavlovic@maths.tcd.ie

Institute of MATHEMATiCS

11000 Belgrade, Yugoslavia

The author is thankful to Professor Marc Rieffel of the University of California, Berkeley, for suggesting this direction of research, and for his further encouragement and discussions. Work on this paper began while the author was a Post-Doctoral Fellow at the Fields Institute for Research in Mathematical Sciences during the $C^{*}$-algebras year 1994/1995, led by Professor George Elliott. It was finished while the author was a Post-Doctoral Fellow at the University of Victoria, Canada, with Professors John Phillips and Ian Putnam, where the research was supported by their NSERC grants. 